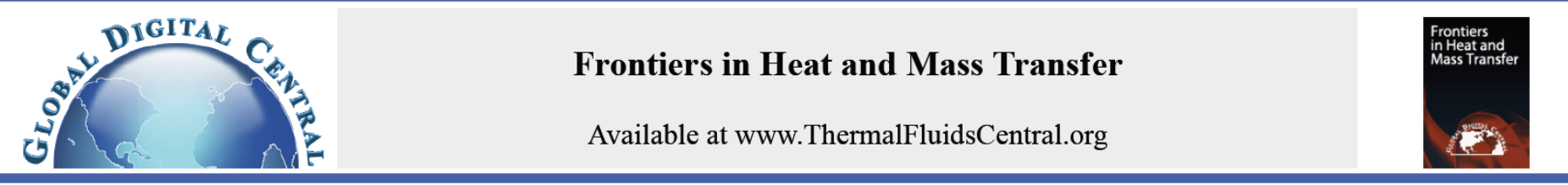

\title{
FLOW SEPARATION IN FALLING LIQUID FILMS
}

\author{
Georg F. Dietze ${ }^{\dagger}$ and Reinhold Kneer \\ Institute of Heat and Mass Transfer, RWTH Aachen University, Aachen, D-52056, Germany
}

\begin{abstract}
Despite the use of liquid films in a wide variety of technical applications involving heat and mass transfer (e.g. nuclear reactors, cooling towers and gas turbines), where they often play an important role, the underlying momentum and heat transport processes within these thin liquid layers remain to be fully elucidated. In particular, this applies to the influence that surface waves, developing due to the film's natural instability, exert on the mentioned processes. In this context, it has been suggested by several experimental and numerical observations that momentum and heat transfer in the capillary wave region (which precedes large surface waves) undergo drastic variations. Indeed, some results have indicated the occurrence of upward flow (i.e. opposed to the gravitational acceleration) in this region. Moreover, evidence of a large increase in wall-side and interfacial transfer coefficients has also been noted. Recently, the authors have established that flow separation takes place in the capillary wave region of 2- and 3-dimensional laminar falling liquid films, partially explaining the above mentioned observations. They showed that the strong change in curvature of the liquid-gas interface in this region causes an adverse pressure gradient (due to the action of surface tension forces) sufficiently large to induce flow detachment from the wall. In the present paper, an in-depth experimental and numerical investigation of this phenomenon in terms of its kinematics and governing dynamics as well as its effect on heat transfer for two different 2-dimensional flow conditions is presented. Experimentally, velocity measurements (using Laser Doppler Velocimetry and Particle Image Velocimetry) and film thickness measurements (using a Confocal Chromatic Imaging technique) were performed in a specifically designed optical test setup. On the numerical side, simulations of the full Navier-Stokes equations as well as the energy equation using the Volume of Fluid (VOF) method were performed. In addition to these investigations, the numerical simulation of a 3-dimensional vertically falling water film, for flow conditions studied in a previous experimental contribution to the literature, was performed. Based on these data, the characteristics of capillary flow separation in the presence of 3-dimensional surface waves were studied. Results show that flow separation takes place in several areas of the resulting complex 3-dimensional capillary wave region, developing multiple separation zones in the shape of vortex tubes. In addition, spanwise flow and an associated eddy induced by the same governing mechanism are shown to occur in this region. This could explain the strong intensification of transfer to 3-dimensional liquid films.
\end{abstract}

Keywords: Falling liquid films, flow separation, heat transfer intensification, three-dimensional waves.

\section{INTRODUCTION}

Falling liquid films, i.e. thin liquid layers (with a thickness of approximately $0.5 \mathrm{~mm}$ ) flowing down an inclined or vertical wall, display a complex free surface topology due to the occurrence of surface waves, which develop as a result of the film's natural instability to perturbations of the liquid-gas interface, Alekseenko et al. (1994). Figure 1 provides an overview of the characteristics of this topology. The central shadowgraph was obtained by Nosoko et al. (1996) and shows the evolution of surface waves on a vertically falling water film excited by way of a controlled disturbance upstream of the liquid inlet. Near the inlet, waves with equally spaced two-dimensional wave fronts are seen to occur, which break-up into 3-dimensional structures further downstream. The sketch on the right side of Figure 1 displays the liquid-gas interface's profile in the 2dimensional region of the flow, consisting of large wave humps preceded by small capillary waves (so called due to the importance of surface tension forces) and separated by a smooth residual layer. The two close-up shadowgraphs on the left side of Figure 1 display typical 3-dimensional surface waves (see also Alekseenko et al. (2005), Petviashvili and Tsvelo- dub (1978)), which are either regular (due to a monochromatic excitation) or irregular (due to the "broad-band" nature of the film's natural instability). In both cases, the large principal wave humps are associated with pronounced 3-dimensional capillary waves.

The current paper provides an in-depth experimental and numerical analysis of the phenomenon of flow separation, taking place in the capillary wave region of falling liquid films, as was established by Dietze et al. (2008, 2009) and Dietze and Kneer (2010). The study considers 2- and 3-dimensional flow conditions, focussing on the case of monochromatically excited waves, i.e. the scenarios on the right side and in the top left corner of Figure 1. A striking feature of this capillary flow separation is the occurrence of backflow (viewed in a wall-fixed coordinate system) within the liquid film. This possibility has been debated in the literature for some time and a summary of relevant works is provided next.

The existence of backflow in falling liquid films was first conjectured by Kapitza (1948) (see also the English translation Kapitza and Kapitza (1965)). Based on an analytical solution to his 2-equation model

${ }^{\dagger}$ Corresponding author. Email: dietze@wsa.rwth-aachen.de 


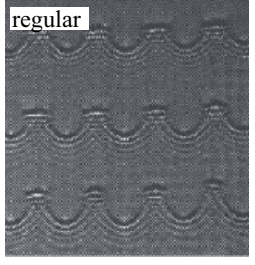

Park and Nosoko (2003), fig. 7d

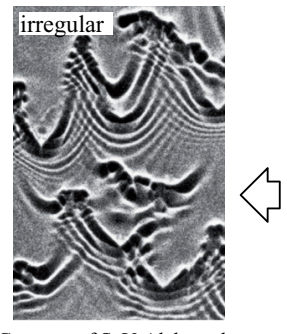

Courtesy of S. V. Alekseenk
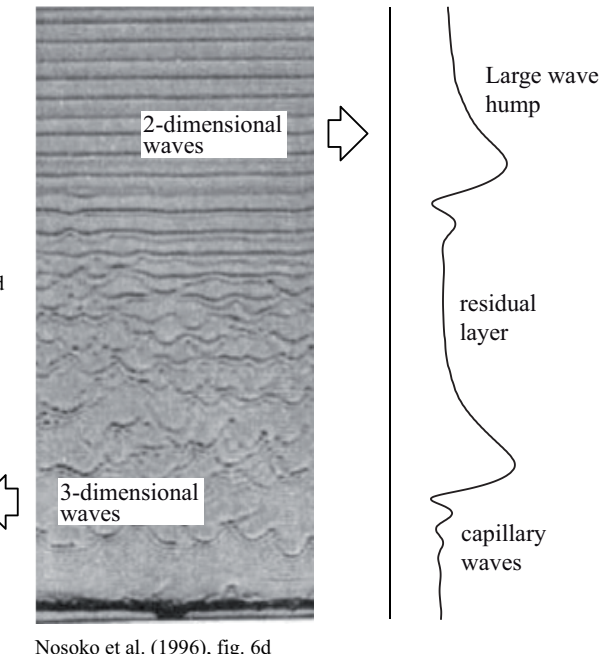

Fig. 1 Free surface topology of 2- and 3-dimensional falling liquid films. Photographs clockwise from top left reproduced with permissions from John Wiley \& Sons, Inc., Elsevier and S. V. Alekseenko (see also Alekseenko et al. (1994)) respectively.

he illustrated the resulting flow field in the film's cross section for the case of harmonic surface waves, as reproduced in Figure 2. Most interestingly, the figure shows a backflow region at the wave trough in the shape of a cellular pattern (this term was later introduced by Malamataris and Balakotaiah (2008)). Although the wave topology investigated by Kapitza (1948) differs substantially from that of Figure 1, a wave trough such as the one in Figure 2 is encountered in principle at all capillary minima. Confirmation of Kapitza's results was provided by Massot et al. (1966), who made a correction to the 2-equation model and introduced a quantitative criterion for the occurrence of backflow. Demekhin et al. (1983) obtained similar results by numerically solving the first order boundary layer equations of film flow. Portalski (1964) tried to provide a physical explanation of the backflow phenomenon, introducing two important terms. Firstly, he stated that "at a fixed point the flow is periodically brought to rest and then reversed by the passage of the wave, so that separation is bound to occur... as in a boundary layer with adverse pressure gradient". Secondly, he introduced the notion of $e d d y$ formation. A first experimental validation of Kapitza's conjecture is contained in the work of Brauer (1956), who observed upward motion of platelets distributed on the film's liquid-gas interface (subsequently designated as interface), whereas liquid phase streamwise velocity data measured by Nakoryakov et al. (1977) exhibit no negative values. However, as pointed out by the authors, their data display a large scatter in the capillary wave region, pointing toward extreme spatio-temporal modulations of the flow field there. Subsequently, Demekhin et al. (1987) investigated one of the regimes of Nakoryakov et al. (1977) numerically (using the boundary layer equations), showing that backflow does take place ${ }^{1}$.

More recent investigations pertaining to backflow considered the falling film in its full complexity, either by numerically solving the NavierStokes equations, or by way of sufficiently resolved measurements. $\mathrm{Nu}-$ merical investigations confirming the existence of backflow in the capillary wave region include those of Salamon et al. (1994), Miyara (1999), Adomeit et al. (2000), Leefken and Renz (2001), Malamataris et al. (2002) and Malamataris and Balakotaiah (2008). Thereby, simulated wave dynamics were validated with experimental data by Adomeit et al. (2000) and Malamataris et al. (2002). In addition, very convincing experimental proof of the backflow phenomenon is contained in the work of Adomeit et al. (2000) in the form of a photograph (recorded with large expo-

\footnotetext{
${ }^{1}$ This was recently confirmed by Malamataris and Balakotaiah (2008) by numerically solving the Navier-Stokes equations for the same case.
}

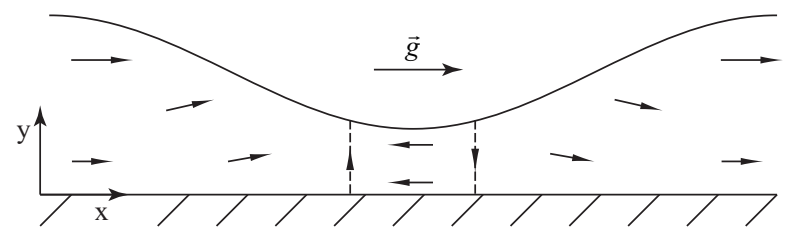

Fig. 2 Illustration by Kapitza (1948) of the flow pattern at the wave trough of a vertically falling liquid film with harmonic surface waves.

sure time) showing loop-shaped pathlines of particles illuminated with a pulsed laser in the capillary wave region.

Recently, Tihon et al. (2003) measured time traces of the local wall shear rate $\partial u /\left.\partial y\right|_{y=0}$ in an inclined film with a large number of capillary waves, exhibiting sign changes (which are associated with flow reversal) at the first two capillary minima. Similar results were obtained by Tihon et al. (2006), where additional numerical simulations corroborated the experimental findings. Moreover, Kunugi and Kino (2005) performed the first full numerical simulation of a falling liquid film with natural 3-dimensional wave dynamics, exhibiting backflow within extensive capillary wave regions. Thereby, the authors conjectured the backflow phenomenon to be responsible for the 3-dimensional instability of 2-dimensional waves ${ }^{2}$, stating that during the transition from 2- to 3dimensional horseshoe-shaped waves, the vorticity vector in the backflow region changes from a spanwise toward a streamwise orientation "like a hair-pin vortex motion". Evidence pointing in this direction is provided in this paper (see subfigure 22(b)).

The characteristics of wall-side heat transfer in 2-dimensional falling liquid films with pronounced capillary waves have been addressed only recently. Adomeit et al. (2000) showed that the heat transfer coefficient $h$ (obtained from numerical simulation) exhibits local maxima at the respective capillary minima, the most significant increase in $h$ occurring at the first capillary minimum. Similar numerical results were obtained by Miyara (2001), Kunugi and Kino (2003) and Kunugi et al. (2005), while experimental confirmation was provided by Schagen et al. (2006). Kunugi and Kino (2005) obtained similar results from their 3 dimensional simulations, reporting a substantial increase of $h$ at the first capillary minimum.

Most recently, Dietze et al. (2008, 2009, 2011) (see also Dietze (2010)) have established that flow separation takes place in the capillary wave region of 2-dimensional laminar falling liquid films, explaining the above mentioned observations of backflow and heat transfer intensification (partially) in the capillary wave region. In the present paper, a coherent account of the different aspects of capillary flow separation, from its kinematics and governing dynamics to its effect on heat transfer, based on experimental and numerical data, is given. Therefore 2 cases of 2-dimensional film flow (an inclined and a vertical film) and one 3dimensional case are considered.

\section{EXPERIMENTAL METHODOLOGY}

A short description of employed experimental methods is provided next. More detailed information can be found in the work of Dietze et al. (2009) as well as Dietze (2010).

In order to access the film cross section with optical velocity and film thickness measurement techniques, a specifically designed optical test setup (see Figure 3) was employed. The principal element of the test setup consists of an elongated cuboid quartz glass body that contains a cylindrical bore creating an inner surface, along which the liquid film develops (see Figure 4). To enable the distortion-free imaging of the film cross section, the refractive indices of working liquid and quartz glass

\footnotetext{
${ }^{2}$ Kunugi and Kino (2005) considered heat transfer and the temperature dependence of fluid properties so that thermal effects such as Marangoni-convection must be taken into account.
} 


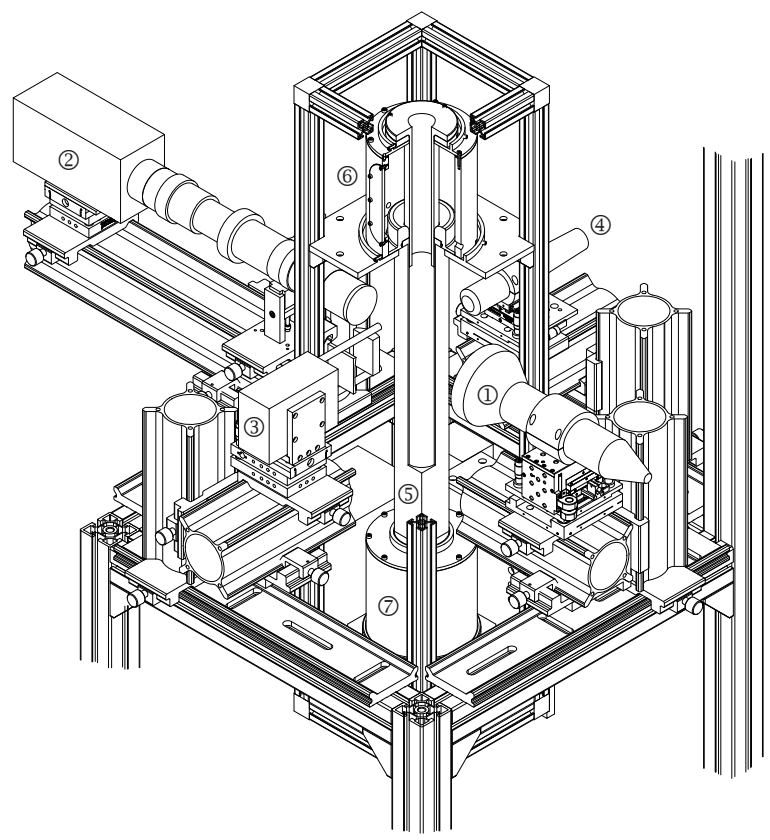

Fig. 3 Optical test setup. 1: LDV-probe; 2: CMOS camera and microscopic lens assembly (PIV); 3: light sheet optics (PIV); 4: CCIprobe; 5: glass body; 6, 7: middle and lower reservoir.

were matched. This was achieved by the choice of an appropriate working liquid (a DMSO-water mixture with $\nu=2.85 \cdot 10^{-6} \mathrm{~m}^{2} / \mathrm{s}, \rho=1098.3$ $\mathrm{kg} / \mathrm{m}^{3}, \sigma=0.0484 \mathrm{~N} / \mathrm{m}$ at $T=25.2^{\circ} \mathrm{C}$ ) and the careful control of the working temperature to $T=25.2{ }^{\circ} \mathrm{C}$.

The optical test setup displayed in Figure 3 is integrated into a standard closed liquid circuit. The working liquid is pumped from the lower reservoir (item 7 in Figure 3) to an upper buffer reservoir (not pictured) situated above the optical test setup by a gear pump. In order to regulate the liquid temperature, a heat exchanger is integrated into the circuit between the lower and upper reservoirs. The volume flow rate was measured with a positive displacement flow meter between the heat exchanger and the upper reservoir. The flow of working liquid leaving the upper reservoir is divided and radially fed to the middle reservoir shown in Figure 3 through two separate entry ports. Upon entering the middle reservoir,

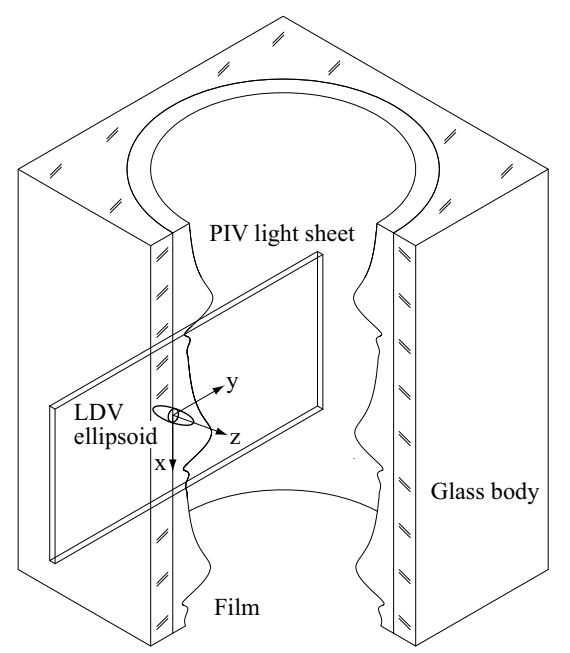

Fig. 4 Sketch of the glass body, illustrating optical access to the falling liquid film's cross section for different velocimetry techniques.

the liquid impinges on a cylindrical overflow the inner surface of which

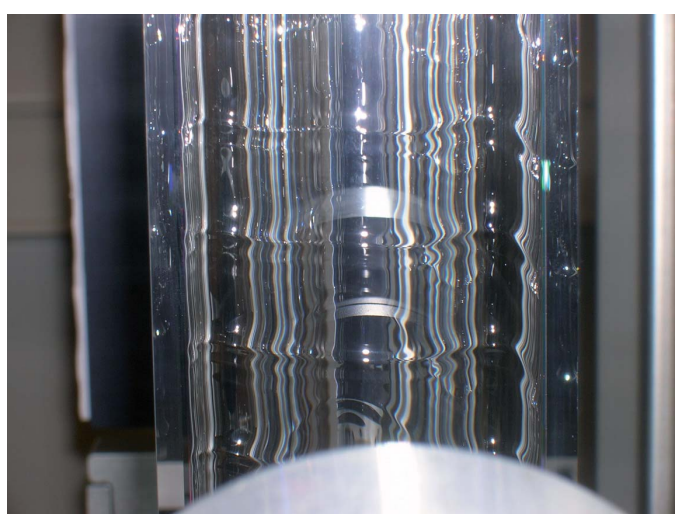

Fig. 5 Photograph of a falling liquid film within the cuboid glass body, exhibiting monochromatically excited 2-dimensional wave fronts.

is rounded, in order to avoid flow separation within the inlet. The actual inlet for the falling liquid film consists of an annulus created between the glass body and a cylindrical insert. The thickness of the annular inlet channel could be varied incrementally through the choice of the insert diameter. During experiments, the temperature of the liquid in the middle reservoir was measured with a resistive thermometer (Pt 100) with an error of $\pm 0.1 \mathrm{~K}$ and the ambient temperature of the laboratory was conditioned to $T=25{ }^{\circ} \mathrm{C}$. The air volume above the liquid level developing in the middle reservoir was connected to an external loudspeaker-driven resonator, enabling the monochromatic excitation of surface waves in the film flow.

Figure 5 shows a photograph of the wavy liquid film in the optical setup for a representative regime with surface waves of relatively large wavelength and precursory capillary waves. This picture shows that wave fronts were 2-dimensional over the entire width of the glass body's face. Under such conditions (detailed operating conditions for the 2-dimensional vertical film investigated experimentally are given in the second line of table 1), simultaneous measurements of streamwise velocity (using Laser Doppler Velocimetry, LDV) and film thickness (using Confocal Chromatic Imaging ${ }^{3}$ or CCI) were performed. Thereby, the LDV measurement ellipsoid was oriented with its main axis in spanwise direction (see Figure 4), yielding a high crosswise spatial resolution. The application of the CCI technique to film thickness measurements in the optical test setup was detailed by Dietze et al. (2009). Particle Image

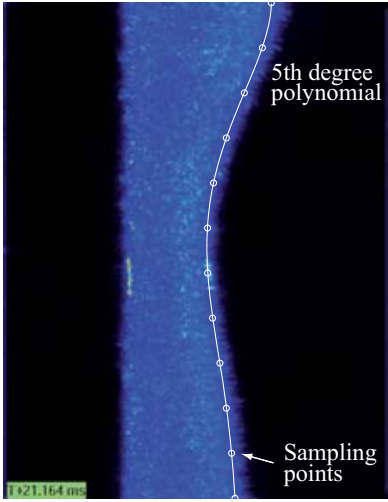

(a) first image: $t=t_{1}$

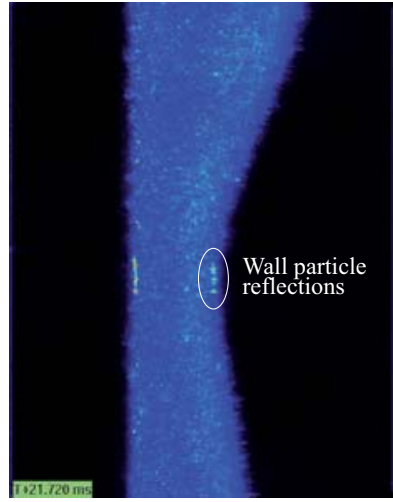

(b) second image: $t=t_{1}+1 / 1800 \mathrm{~s}$
Fig. 6 Successive digital PIV images recorded with the CMOS camera in the region of the first capillary minimum.

\footnotetext{
${ }^{3}$ See Cohen-Sabban et al. (2001) for a description of the measurement principle.
} 


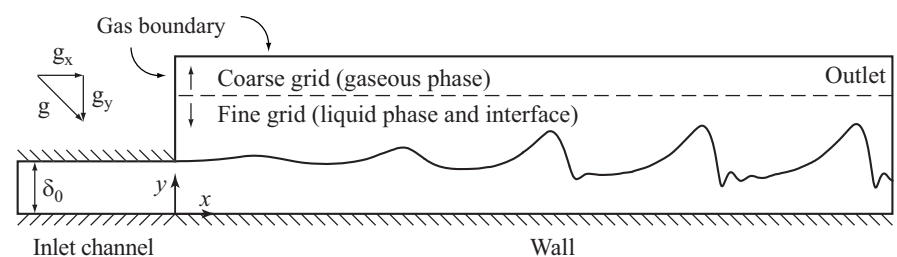

Fig. 7 Computational domain used for the 2-dimensional simulations.
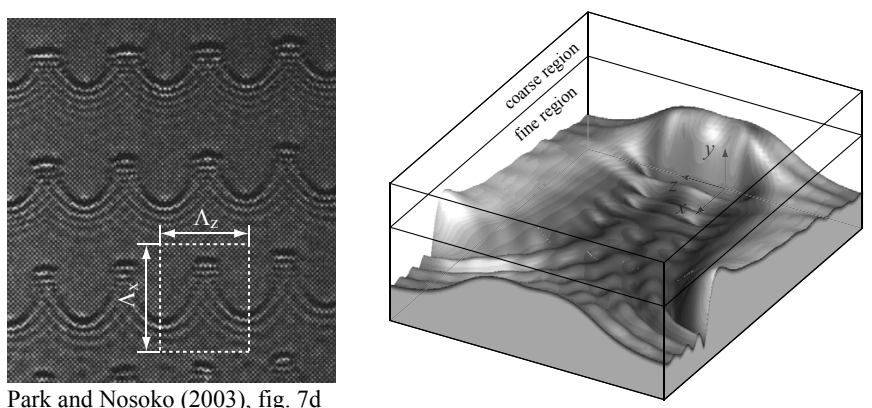

Fig. 8 Shadowgraph of the 3-dimensional film (with permission from John Wiley \& Sons, Inc.), demarcating the periodic wave segment included in the computational domain. $\Lambda_{x}=25 \mathrm{~mm}, \Lambda_{z}=20 \mathrm{~mm}$.

Velocimetry (PIV) was employed to measure the velocity field within the liquid phase, using a CMOS camera for recording (Figure 6 shows a sample image pair) and a pulsed laser light sheet for illumination (see Figure 4).

\section{NUMERICAL METHODOLOGY}

Numerical results presented in this paper are based on two 2-dimensional simulations as well as one 3-dimensional simulation (for experimental conditions from Park and Nosoko (2003)) of falling liquid film flow. In all cases, the Volume of Fluid method (see Hirt and Nichols (1981)) in conjunction with the Continuum Surface method (see Brackbill et al. (1992)) was employed to solve the Navier-Stokes, continuity and energy equations.

Methodological details concerning the respective simulations can be found in the works of Dietze et al. $(2008,2009,2011)$ as well as Dietze (2010). Operating conditions for the respective simulations are specified in table 1. The computational domain employed for 2-dimensional simulations is sketched in Figure 7. Liquid enters the domain from the left through an inlet channel bounded by two parallel walls. In order to excite surface waves, a monochromatic disturbance of frequency $f$ was imposed on the velocity profile at the liquid inlet. After leaving the inlet channel at $x=0$, the liquid enters the 2-phase region of the computational domain, developing surface waves. An extensive validation of 2-dimensional numerical simulations based on LDV and CCI measurements (as well as grid dependence analysis) was performed by Dietze (2010) and a comparison for the vertical film considered in this paper is shown in Figure 12.

Table 1 Flow conditions for the investigated falling liquid films.

\begin{tabular}{cccccc}
\hline Case & $R e$ & $K a$ & $W e$ & $\varphi(\operatorname{deg})$ & $f(1 / \mathrm{s})$ \\
\hline Inclined & 15.6 & 133.6 & 2.0 & 35 & 15.0 \\
Vertical & 15.0 & 509.5 & 8.0 & 90 & 16 \\
3-D & 59.3 & 3940.2 & 6.3 & 90 & $\Lambda_{x}=25 \mathrm{~mm}$ \\
\hline
\end{tabular}

As opposed to the 2-dimensional simulations, the performed 3-dimensional simulation does not represent the complete spatial evolution of the liquid film, the computational domain being restricted to one segment of the periodic 3-dimensional wave pattern, as illustrated in Figure 8 . The length and width of the domain were accordingly set to the streamwise and spanwise wave lengths $\Lambda_{x}$ and $\Lambda_{z}$, which were obtained from Park and Nosoko (2003); Scheid et al. (2006). Liquid and gas enter and leave the computational domain through streamwise periodic boundaries permeable for both phases. In z-direction, the domain is limited by symmetric boundaries, imposing spanwise symmetry.

\section{TWO-DIMENSIONAL CAPILLARY FLOW SEPARATION - KINEMATICS -}

A visualization of capillary flow separation as it occurs in the 2-dimensional inclined and vertically falling liquid films is provided in figures 9 and 10 respectively. These display the spatio-temporal evolution of simulated liquid phase streamlines at the first capillary minimum. A pictogram of the corresponding streamwise film thickness distribution, highlighting the region depicted in the streamline plot with an arrow, is also included in each subfigure. This highlighted region moves downstream with wave celerity, tracking the first capillary minimum of a single wave during its entire development.

Both streamline plot sequences exhibit the same behaviour in the initial stages of wave development. As the first capillary minimum takes shape, streamlines underneath it distort into a pattern typically associated

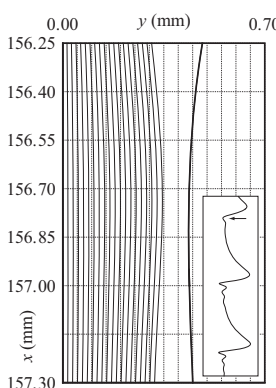

(a) $t=1.16055 \mathrm{~s}$

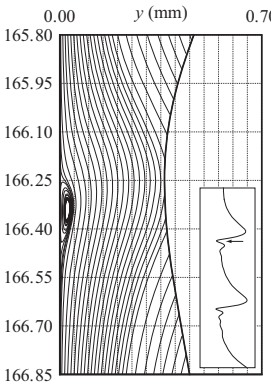

(d) $t=1.19325 \mathrm{~s}$

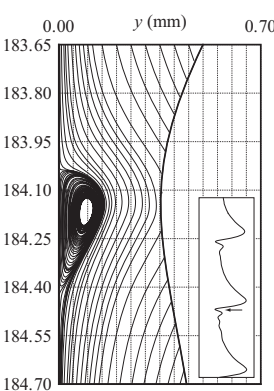

(g) $t=1.25062$

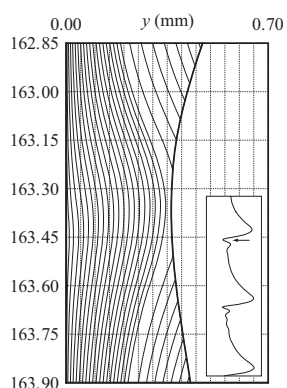

(b) $t=1.18350 \mathrm{~s}$

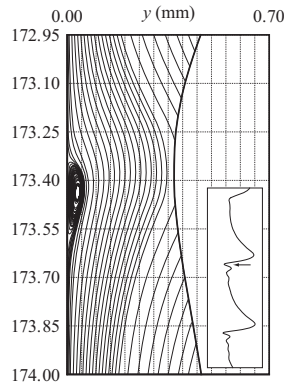

(e) $t=1.21620$

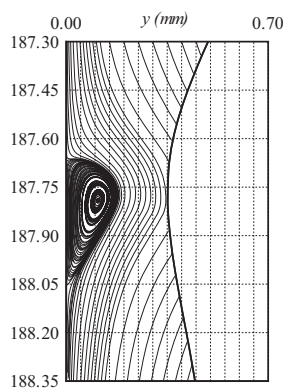

(h) $t=1.26210 \mathrm{~s}$

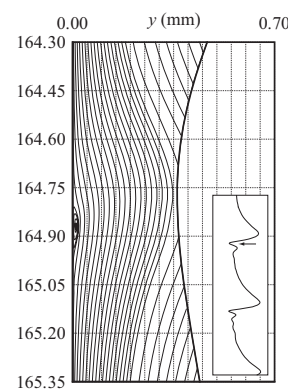

(c) $t=1.18830 \mathrm{~s}$

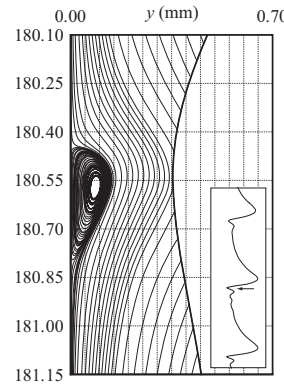

(f) $t=1.23915 \mathrm{~s}$

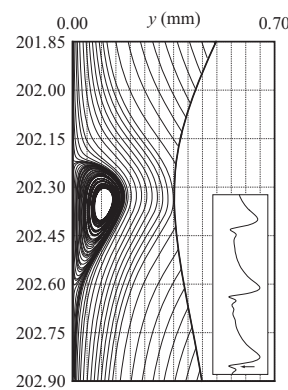

(i) $t=1.30800 \mathrm{~s}$
Fig. 9 Temporal evolution of simulated streamlines at the first capillary minimum for the inclined film. 


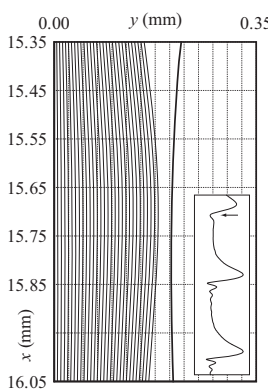

(a) $t=2.95750 \mathrm{~s}$

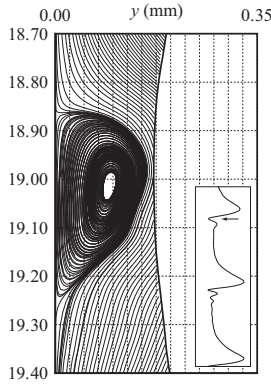

(d) $t=2.97000 \mathrm{~s}$

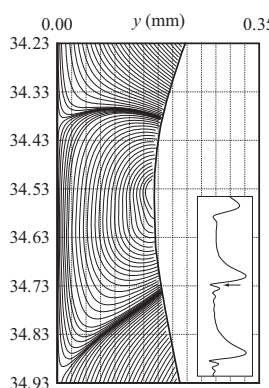

(g) $t=3.02000 \mathrm{~s}$

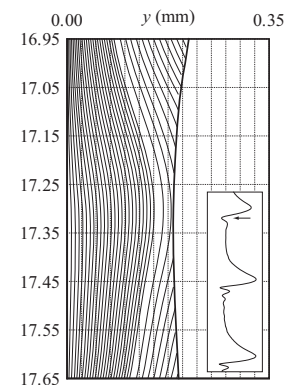

(b) $t=2.96375 \mathrm{~s}$

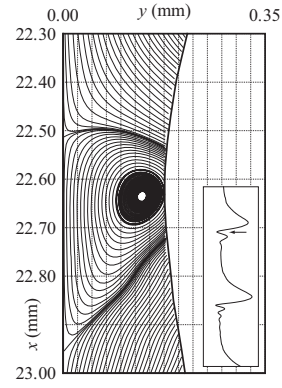

(e) $t=2.98250 \mathrm{~s}$

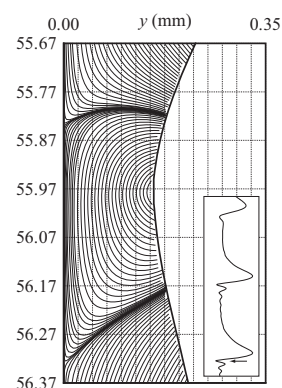

(h) $t=3.08350 \mathrm{~s}$

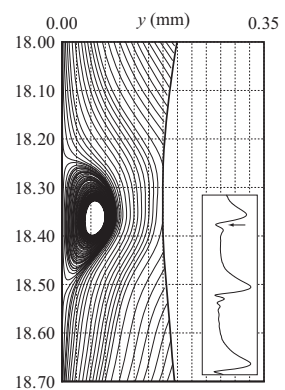

(c) $t=2.96750 \mathrm{~s}$

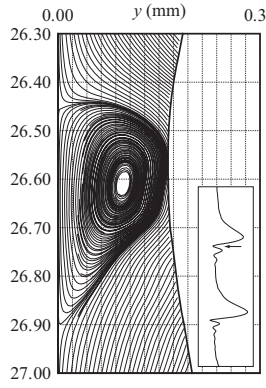

(f) $t=2.99500 \mathrm{~s}$

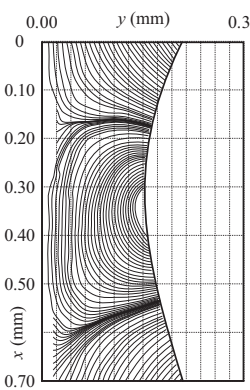

(i) PIV data
Fig. 10 Temporal evolution of simulated and experimental (subfigure (i)) streamlines at the first capillary minimum for the vertical film.

with decelerated and re-accelerated flow. Indeed, tracking a streamline in streamwise direction, the latter moves away from the wall while approaching the capillary minimum and re-converges toward it after passing the minimum. This streamline distortion grows more pronounced as the capillary waves develop, until the flow separates by detaching from and subsequently re-attaching to the bounding wall (see subfigures 9(c), 9(d) and 10(c)) similar to "classical" flow separation. In the process, a Capillary Separation Eddy (CSE) is formed, which subsequently grows until it attains a constant size once the capillary wave topology is fully developed.

In this respect, a significant difference between the inclined and vertical film is evident. In the first case, the CSE stays fully contained in the liquid phase (see Figure 9(i)), assuming a crosswise size of approximately half the minimal film thickness. In the second case, the CSE outgrows the liquid film, assuming an open shape with streamlines ending at the liquid-gas interface (see subfigure 10(h)). The possibility of such a scenario was in principle established by Rood (1994), who investigated vortex interactions with a free surface and stated that, when a vortex breaks up and "attaches" to a free surface, interfacial vorticity transport takes place (a discussion of vorticity transport within the CSE was provided by Dietze et al. (2009)). Interestingly, the streamline pattern in subfigure 10(h) confirms the flow structure conjectured by Kapitza (1948) (see Figure 2). An experimental confirmation is provided in sub-

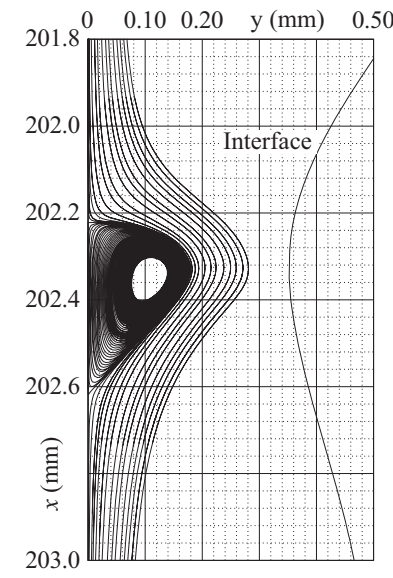

(a) Inclined film: streamlines

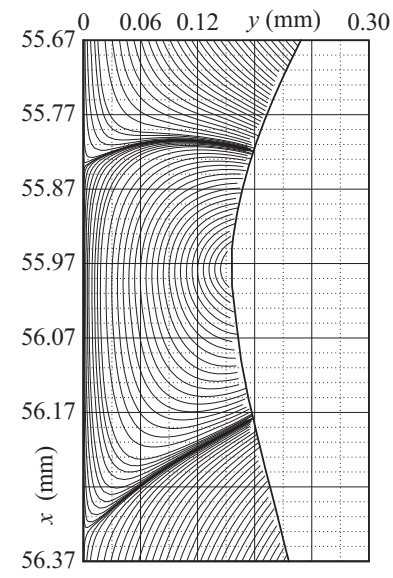

(c) Vertical film: streamlines

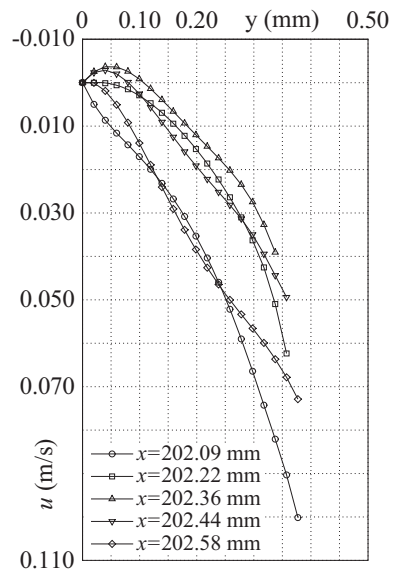

(b) Inclined film: velocity profiles

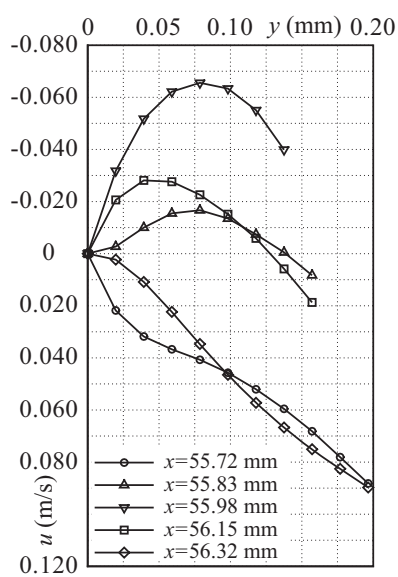

(d) Vertical film: velocity profiles
Fig. 11 Simulated streamlines and streamwise velocity profiles at the first capillary minimum for the inclined and vertical film.

figure 10(i) in the form of streamlines computed from PIV data measured in the optical test setup (see Dietze et al. (2009) for further experimental results). Moreover, subfigures $10(\mathrm{e})$ to $10(\mathrm{~g})$ show that, before attaining its final open shape (subfigure 10(h)), the CSE reverts back to a closed shape (subfigures 10(e) and 10(f)), suggesting a two-way coupling between flow separation and capillary wave dynamics (similar observations were recently made by Malamataris and Balakotaiah (2008)). Consequently, it can be envisaged that capillary flow separation may play a role in the (secondary) instability of wavy liquid films.

Figure 11 shows streamwise velocity profiles within the developed CSE for the two 2-dimensional cases. These exhibit flow reversal, as well as a streamwise sign change of their near-wall curvature similar to "classical" flow separation. From the point of view of multiple-equation wave dynamics models (see Scheid et al. (2006); Mudunuri and Balakotaiah (2006); Nguyen and Balakotaiah (2000) and Ruyer-Quil and Manneville (2000)), that approximate the streamwise velocity distribution with polynomials, this means that the latter should be at least of third order, which is not the case for lowest order models. Comparing profiles in subfigures 11(b) and 11(d), it is clear that much stronger backflow occurs for the vertical film. Indeed, the velocity profile passing through the center of the open CSE ( $x=55.98 \mathrm{~mm}$ ) exhibits a maximal negative value approximately ten times larger than the corresponding profile for the inclined liquid film. At this position, a considerable volume flux per unit width of 

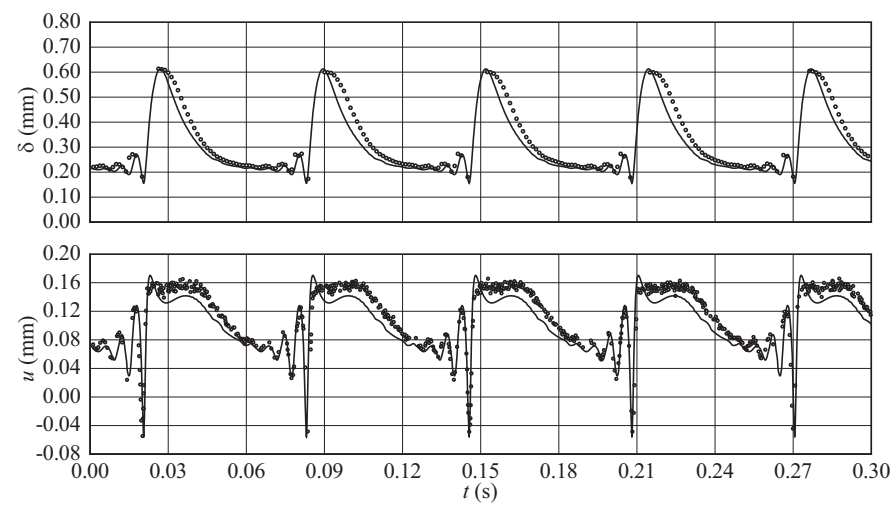

(a) $y=120 \mu \mathrm{m}$

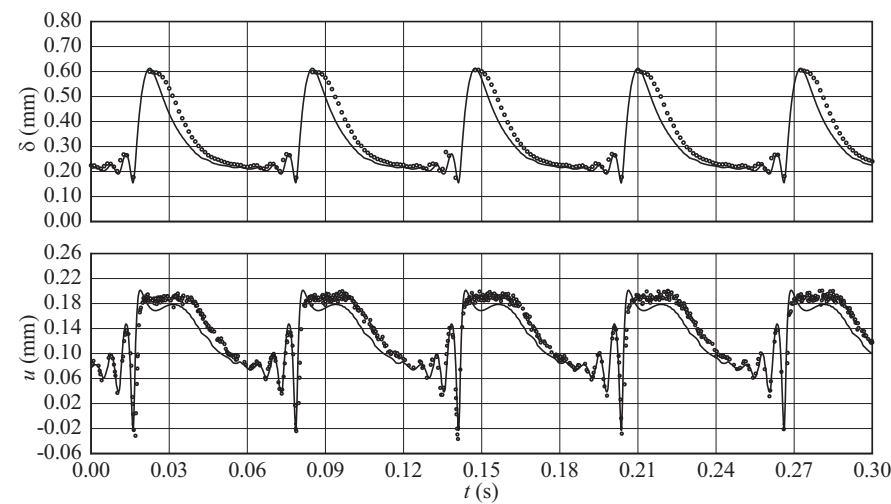

(b) $y=160 \mu \mathrm{m}$

Fig. 12 Film thickness (top) and streamwise velocity (bottom) time traces for the vertical film. Circles: experiment (CCI and LDV); lines: simulation

approximately $3 \cdot 10^{-6} \mathrm{~m}^{2} / \mathrm{s}$, yielding a local Reynolds number value of approximately 1 , flows counter to the gravitational acceleration.

Finally, Figure 12 depicts streamwise velocity and film thickness time traces obtained from experiments (circles) and numerical simulation (lines) for the 2-dimensional vertical film. Data were evaluated at two different crosswise positions within the thickness of the residual layer. They clearly show flow reversal at the first capillary minimum as well as a strong correlation between film thickness and streamwise velocity.

\section{TWO-DIMENSIONAL CAPILLARY FLOW SEPARATION - GOVERNING DYNAMICS -}

From elaborations concerning the kinematics of capillary flow separation it is clear that this phenomenon is quite similar to "classical" separation of wall-bounded single-phase flows (see e.g. Prandtl (1961); Sychev (1998)) at least in the early stages of its development. This also holds for the developed state of the phenomenon if the CSE stays contained within the liquid film's residual layer. If however, the CSE outgrows the local film thickness, this similarity is no longer valid and considerably different kinematics take hold.

An even more important difference with respect to "classical" flow separation concerns the governing dynamics of capillary flow separation. While the driving cause in the former case is an adverse pressure gradient imposed by the external flow, falling liquid films considered here flow in a largely quiescent gaseous atmosphere, which is virtually unbounded on the far-field side. Consequently, the driving cause for capillary flow separation cannot stem from the external gaseous phase.

The occurrence of flow separation in falling liquid films, especially in the case of a vertical arrangement, is at first unexpected, since this
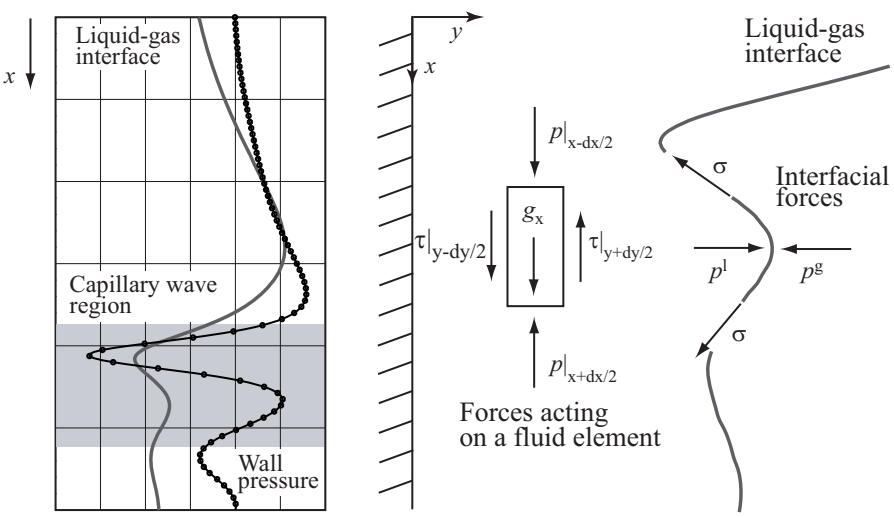

Fig. 13 Illustration of the relation between interface topology and liquid phase static pressure.

means that liquid flows in the direction opposite to gravity, which accelerates the film downward in the first place. Thus, the principal question to be answered concerns the force causing such flow reversal. Obviously, this force must act in upstream direction. Considering that the wall parallel component of the gravitational force acts in downstream direction and that viscosity forces are inner forces, only the resulting pressure force on a fluid element can be retained as $\operatorname{such}^{4}$ (see the illustration on the right hand side of Figure 13). More precisely, since capillary flow separation initiates at the bounding wall, it is the wall distribution of static pressure, that is of principal interest. Accordingly, one can conjecture that capillary flow separation must be caused by a positive streamwise wall pressure derivative occurring in the capillary wave region.

The mechanism generating this positive pressure derivative can be understood by considering the relation between liquid phase static pressure and interface topology. This is given by the normal coupling condition between liquid and gaseous phase (see e.g. Alekseenko et al. (1994)), which can be simplified assuming the dominance of capillary and pressure forces in the capillary wave region:

$$
\frac{\partial p_{\mathrm{w}}}{\partial x} \approx \frac{\partial p_{1}}{\partial x} \approx-\sigma \frac{\partial \kappa}{\partial x}, \quad \kappa=\frac{\partial^{2} \delta / \partial x^{2}}{\left(1+(\partial \delta / \partial x)^{2}\right)^{3 / 2}},
$$

where $\delta$ designates the film thickness, $p_{1}$ the liquid-side interfacial pressure and $\kappa$ the interface curvature. Thereby, as established by Malamataris et al. (2002), the distribution of interfacial pressure is, to a large extent, imposed on the wall pressure $p_{\mathrm{w}}$. The physical mechanism underlying equation (1) results from the interfacial pressure jump caused by tensile forces, as illustrated on the right hand side of Figure 13. This pressure jump (from liquid to gas) must act in opposition to tensile forces to maintain the interface's distortion. Consequently, it is negative in a wave hump and positive in a wave trough. Neglecting pressure variations in the gaseous phase, this means that liquid-side interfacial pressure (and therewith wall pressure) increases from wave trough to wave hump. This yields a positive (or adverse) streamwise pressure derivative, i.e. a resulting pressure force directed upstream. At the same time, the streamwise change in interface curvature from positive in the wave trough to negative in the wave hump is negative, which is in accordance with equation (1).

In order to enable flow separation, the resulting adverse pressure force must surmount the downward gravitational force, yielding:

$$
-\frac{\partial p_{\mathrm{w}}}{\partial x} \approx \sigma \frac{\partial \kappa}{\partial x}>\rho g_{x} \Rightarrow \Psi=\frac{-\partial p_{\mathrm{w}} / \partial x}{\rho g_{x}}<-1,
$$

where the force ratio $\Psi$ was introduced. Subfigures 14(b) and 14(d) depict streamwise distributions of $\Psi$ for the inclined and vertically falling liquid film respectively. From these it is evident that the condition for flow

\footnotetext{
${ }^{4}$ Excluding soluto-capillary and thermo-capillary forces.
} 


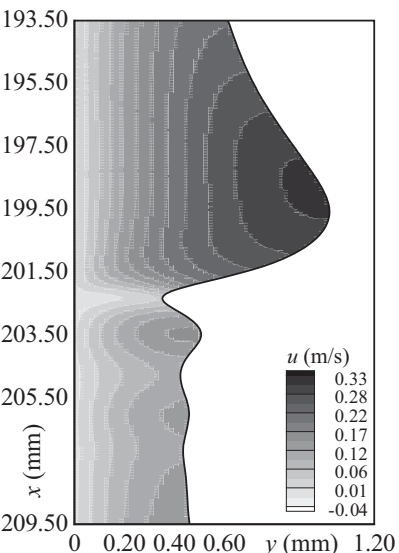

(a) Inclined film

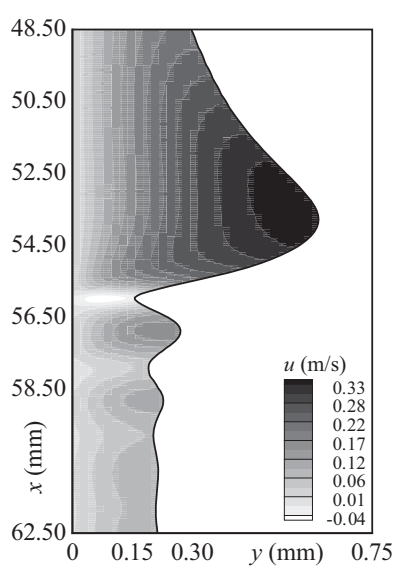

(c) Vertical film

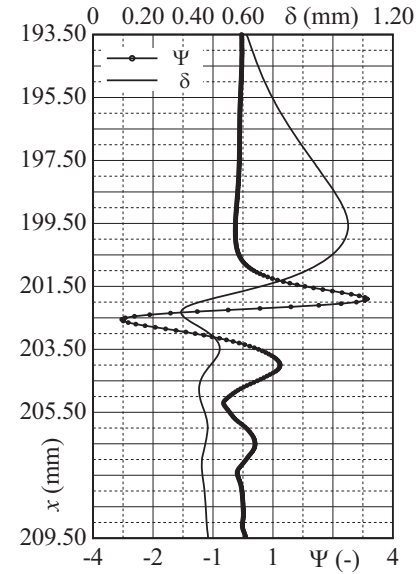

(b) Inclined film: force ratio $\Psi$

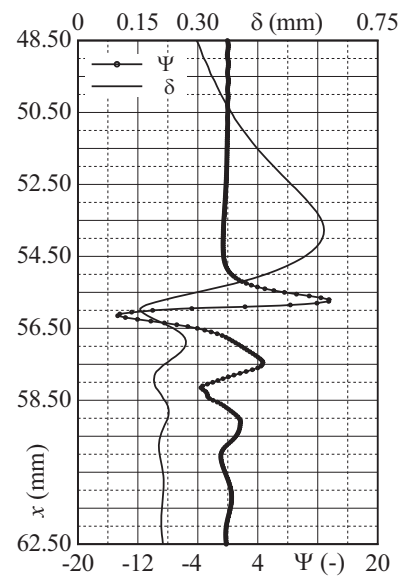

(d) Vertical film: force ratio $\Psi$
Fig. 14 Streamwise velocity contours (left) and force ratio $\Psi=$ $-\partial p /\left.\partial x\right|_{y=0} /\left(\rho g_{x}\right)$ for the inclined and vertical film.

reversal is fulfilled at the first capillary minimum in both cases. Further $\Psi$ attains much larger negative values (meaning a larger upward driving force) for the vertical film, which is in accordance with the stronger flow reversal evidenced in subfigure 11(d).

\section{TWO-DIMENSIONAL CAPILLARY FLOW SEPARATION - EFFECT ON HEAT TRANSPORT -}

Subfigures 15(a) and 15(b) depict temperature contours within a single wave in the fully developed region of the inclined $(\operatorname{Pr}=66.5)$ and vertical $(P r=30.6)$ film, as obtained from wall-side heat transfer simulations performed for constant wall and inlet temperatures $T_{\mathrm{w}}=350 \mathrm{~K}$ and $T_{0}=300$ K. Corresponding wall Nusselt number $\left(N u=h \delta_{\mathrm{Nu}} / k\right)$ time traces are illustrated in subfigures 15 (c) and 15(d). In both representations it is evident that heat transfer is intensified at the first capillary minimum (in accordance with the previously mentioned works of Adomeit et al. (2000); Miyara (2001); Schagen et al. (2006)). Bearing in mind the kinematics of capillary flow separation occurring in this region, it is reasonable to assume that the underlying cause is the CSE-induced intensification of crosswise convective transport in the liquid phase. Based on an Eulerian approach one could assume that the mixing length $l$ characterizing this convective transport is of the order of the CSE's size. However, this approach does not account for the fact that the wave celerity is larger than the mean flow velocity in the residual layer, meaning that surface waves repeatedly pass over fluid elements traveling there. Consequently,

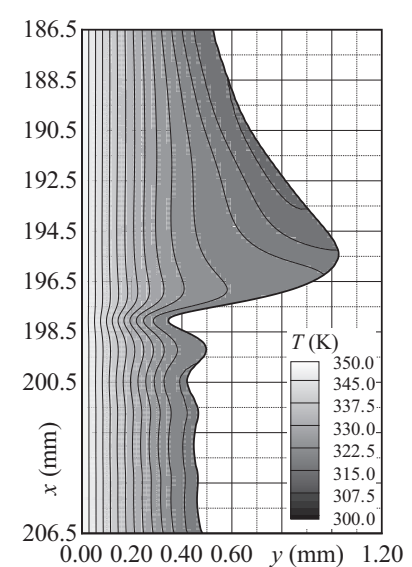

(a) Inclined film

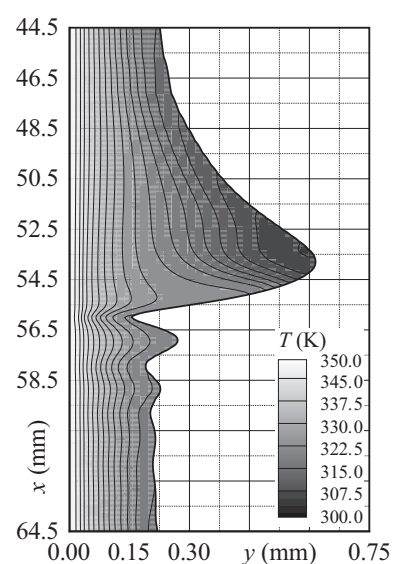

(b) Vertical film

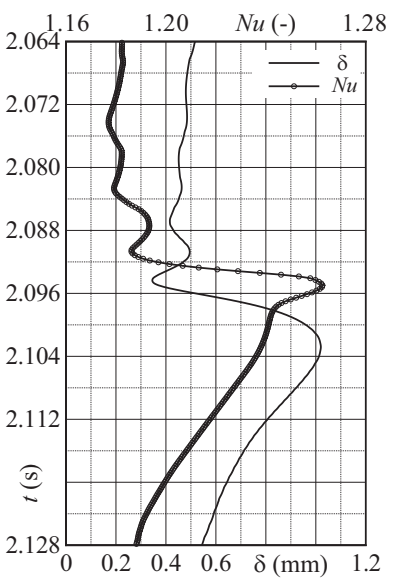

(c) Inclined film: $x=198.0 \mathrm{~mm}$

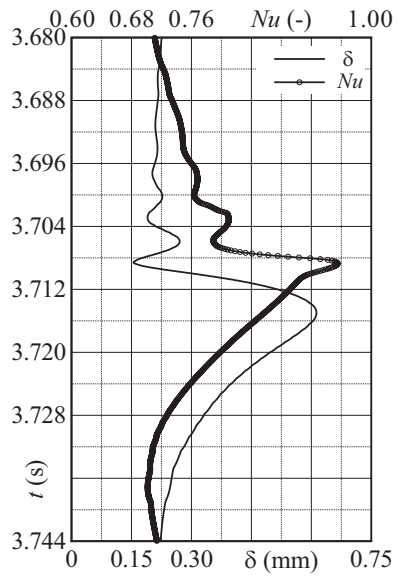

(d) Vertical film: $x=56.0 \mathrm{~mm}$
Fig. 15 Simulated temperature contours and time traces of Nusselt number and film thickness in the fully developed region for the inclined and vertical film.

the latter are subjected to the CSE's kinematics only for a more or less short transition period. Thus, a Lagrangian view seems to be more adequate. This is adopted in figures 16 and 17, which depict simulated fluid element pathlines in the residual layer for the inclined and vertical film respectively. In both figures, the first two subfigures display the state of the pathlines at two different time points during the transition of the first capillary minimum. In addition, subfigures 16(c) and 17(c) depict the pathlines after the passage of the first capillary minimum. Finally, subfigures $16(d)$ and 17 (d) provide a close-up view of pathlines near the wall. From these pathline sequences several general conclusions pertaining to both cases can be drawn. First, the CSE clearly induces crosswise convective transport as it passes over a considered position. Thereby, fluid elements move toward the wall and away again, their pathlines exhibiting "hook"- or "loop"-shaped distortions depending on whether or not they encounter flow reversal. Second, the mixing length $l$ induced by these distortions increases with wall distance, owing to the fact that fluid elements traveling further from the wall exhibit larger streamwise velocities and are thus exposed over a longer time span to the capillary waves passing their position. This effect can be expressed by the simple relation:

$$
l=\frac{1}{2} \tilde{v} \Delta t \approx \frac{1}{2} \tilde{v} \frac{\Lambda_{\mathrm{c}}}{(c-u)},
$$

where $\tilde{v}$ designates the characteristic crosswise velocity induced by the CSE, $c$ signifies the wave celerity and $u$ the local streamwise velocity 


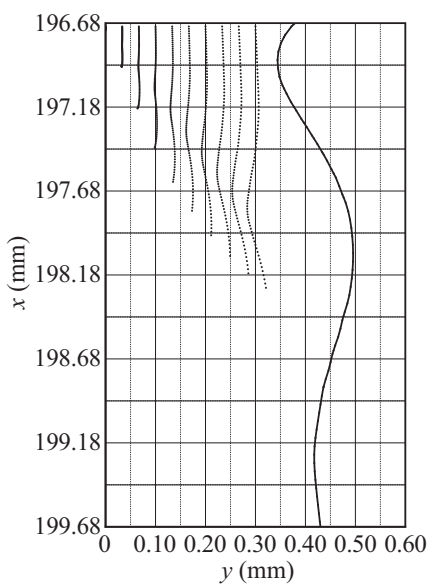

(a) $t=2.090925 \mathrm{~s}$

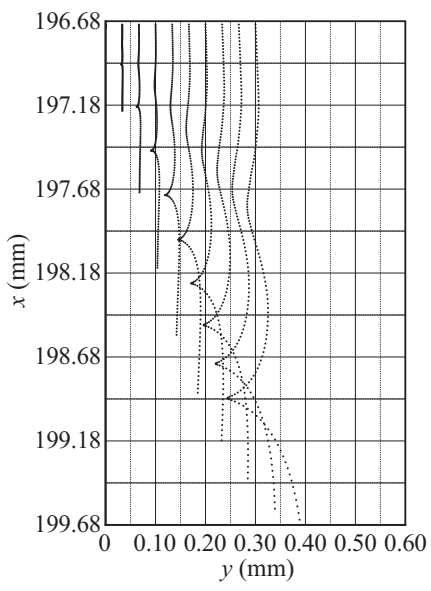

(c) General view

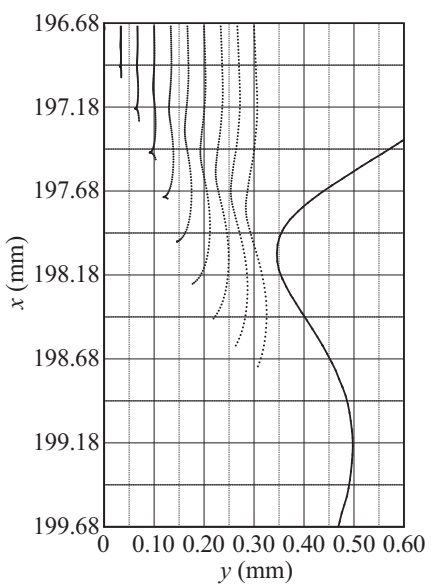

(b) $t=2.094525 \mathrm{~s}$

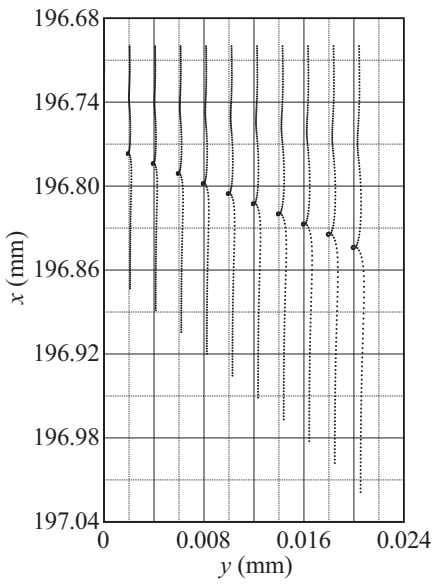

(d) Close-up view

Fig. 16 Simulated fluid element pathlines for the inclined film.

component prior to the capillary wave transit (which can be assumed as semi-parabolic), while $\Lambda_{\mathrm{c}}$ is the capillary wave length.

In terms of a comparison between the inclined and vertical film, the following conclusions can be drawn. Owing to the fact that the CSE in the inclined film is closed, "loop"-shaped pathline distortions are only encountered very close to the wall, whereas for the vertical case they occur over the entire minimal film thickness. In subfigure 17(c), a photographic insert is included, which shows a "loop"-shaped pathline obtained experimentally by Al-Sibai (1998) under somewhat different flow conditions using a similar experimental setup (developed by Adomeit and Renz (2000)). Secondly, the mixing length induced by the CSE in the vertical case is significantly larger, which can be attributed to the larger characteristic crosswise velocity $\tilde{v}$ induced by the open CSE. However, the Nusselt number time traces in subfigures 15 (c) and 15(d) do not exhibit a significantly larger CSE-induced increase for the vertical case. This in turn can be explained by the crosswise decrease in mixing length with decreasing wall distance (as formulated in equation (3)), which yields very small values of $l$ close to the wall for both cases. Finally, judging from the very large pathline distortions near the interface, it can be inferred that interfacial heat transfer is affected to a much larger extent by the CSE, which would confirm previous observations by Seban and Faghri (1978); Alekseenko et al. (1994); Yoshimura et al. (1996); Rastaturin et al. (2006).

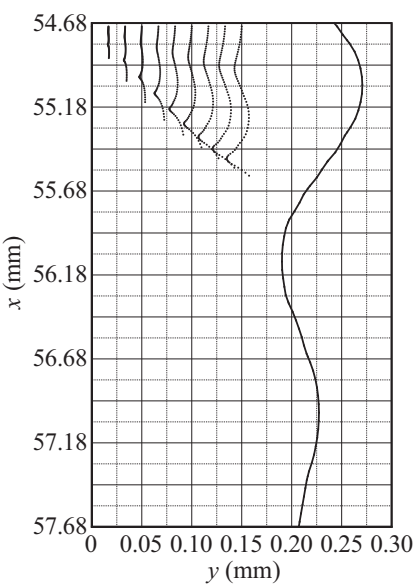

(a) $t=3.265650 \mathrm{~s}$

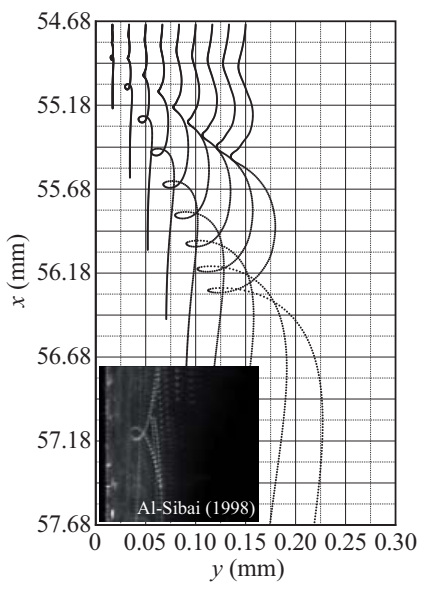

(c) General view

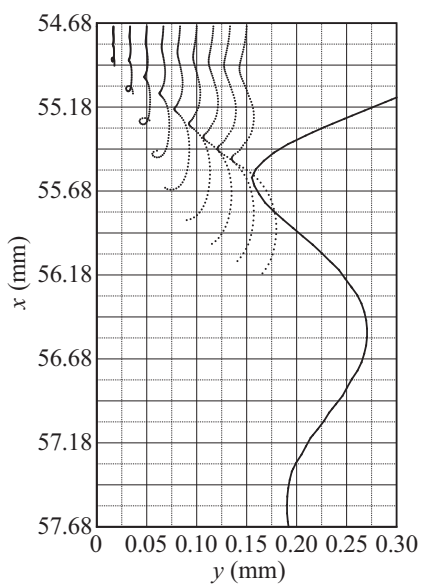

(b) $t=3.269925 \mathrm{~s}$

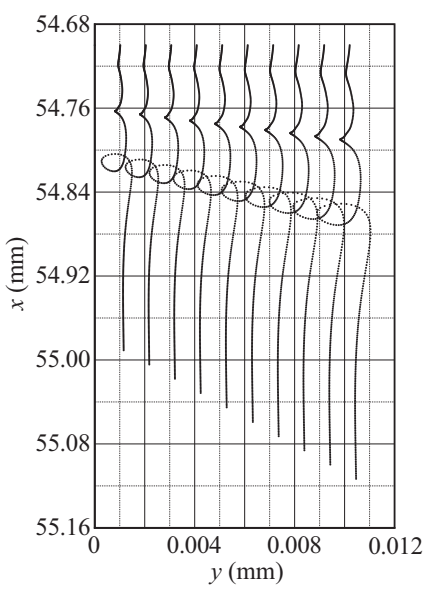

(d) Close-up view
Fig. 17 Simulated fluid element pathlines for the vertical film.

\section{THREE-DIMENSIONAL CAPILLARY FLOW SEPARATION}

Figure 18 represents the fully developed interface topology for the 3dimensional simulation over one streamwise wavelength and three spanwise wavelengths. Similar to the 2-dimensional case, the interface is characterised by large wave humps preceded by several capillary waves of considerable amplitude. However, in contrast to the 2-dimensional case, wave fronts of large wave humps are distorted into horseshoe-like shapes. At the position where two horseshoe-shaped wave fronts meet, a large intermediate hump, representing the global film thickness maximum, occurs. As a further consequence of the horseshoe-shaped wave fronts, capillary waves preceding two adjacent large wave humps interfere with one another. The resulting region of capillary interference is characterised by capillary wave humps and troughs alternating in streamwise and spanwise direction in the form of a checkerboard or herringbone pattern (these terms were previously introduced by Chang et al. (1994) and Liu et al. (1995) respectively to describe the interface topology of interacting 3-dimensional large waves.). Thereby, capillary waves preceding the large intermediate hump are particularly pronounced.

A comparison of the computed wave topology with the corresponding experimental shadowgraph from Park and Nosoko (2003) is shown in Figure 19. The numerical simulation reproduces several key features of the experimental shadowgraph. Firstly, the horseshoe-shaped form of the main wave humps is more or less well captured. Secondly, the number of capillary waves preceding these wave humps as well as the pattern produced by their interference is reproduced. Finally, the two-dimensional 


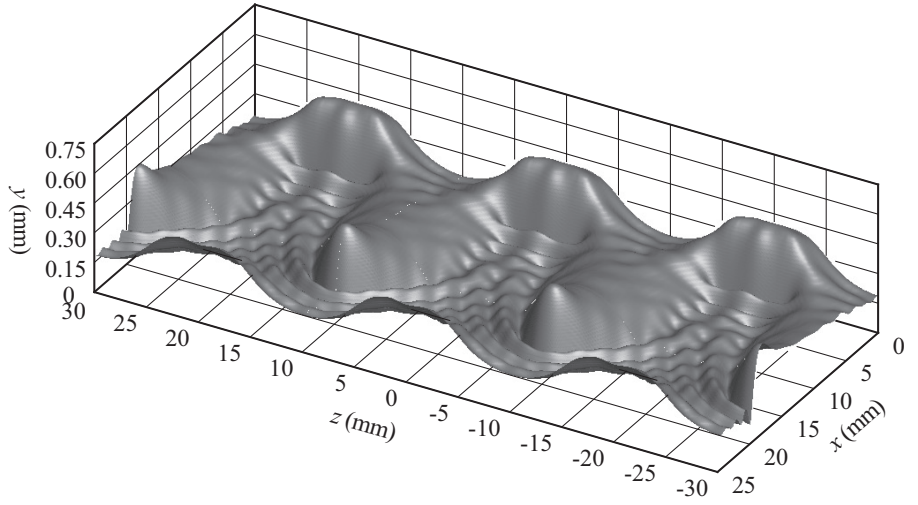

Fig. 18 Interface topology of the simulated 3-dimensional falling liquid film (three spanwise wavelengths): $R e=59.3, K a=3940.2$.

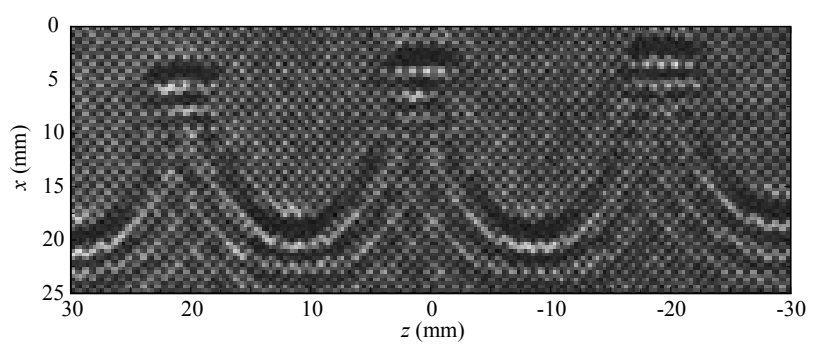

(a) Figure 7d from Park and Nosoko (2003)

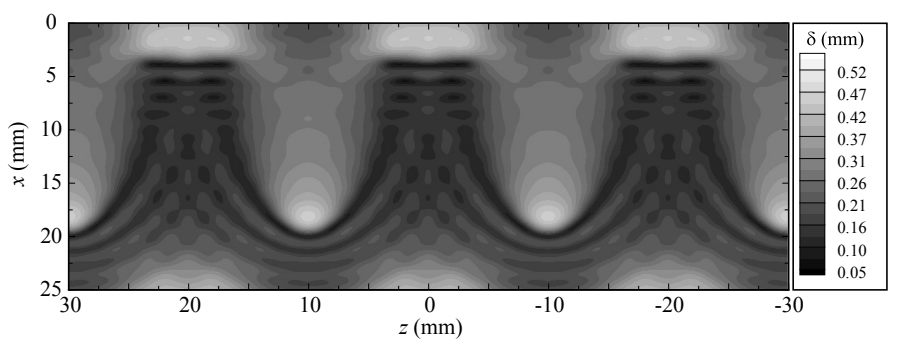

(b) Numerical simulation

Fig. 19 Comparison of numerical and experimental wave topology for the 3-dimensional film: $R e=59.3, K a=3940.2$. Top figure reproduced with permission from John Wiley \& Sons, Inc.

nature of the intermediate hump and its preceding capillary waves is obtained, the lateral extension of these intermediate wave fronts also corresponding reasonably well.

Based on the 3-dimensional representation of wave topology in Figure 18 and the corresponding top view of the film thickness distribution in subfigure 19(b), it can be stated that a large part of the considered film's interface is affected by capillary wave dynamics. In order to assess their influence on the liquid phase flow field, subfigures 20(b) and 20(c) depict contours of the streamwise $\tau_{\mathrm{w} x}=\mu \partial u /\left.\partial y\right|_{y=0}$ and spanwise $\tau_{\mathrm{w} z}=\mu \partial w /\left.\partial y\right|_{y=0}$ wall shear stress, corresponding to the representation of film thickness contours in subfigure 19(b). The contour levels in subfigure 20(b) are chosen such that only negative values of $\tau_{\mathrm{w} x}$ are associated with gray scales other than white, allowing to identify regions of flow reversal. Accordingly, capillary flow separation is seen to occur in three distinct regions.

At the first capillary minimum, flow separation is seen to occur over almost the entire spanwise wavelength. Thereby, in front of the intermediate hump, the streamwise wall shear stress attains the largest negative values, indicating strong backflow, as is expected from the large interface

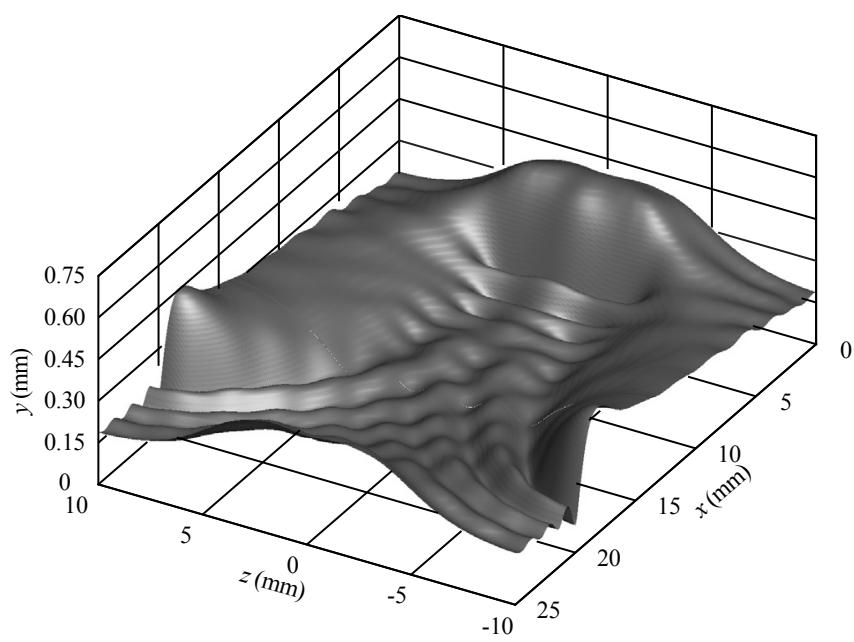

(a) Free surface topology (single spanwise wavelength)

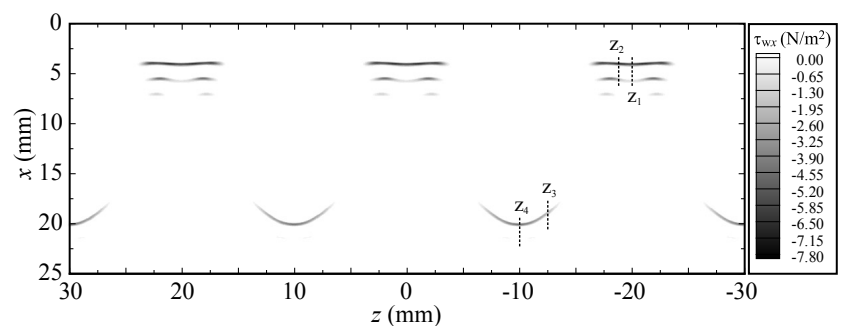

(b) Streamwise wall shear stress: $\tau_{\mathrm{w} x}=\mu \partial u /\left.\partial y\right|_{y=0}$

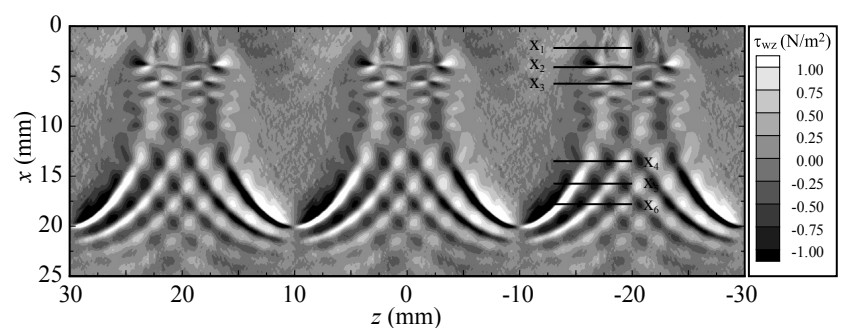

(c) Spanwise wall shear stress: $\tau_{\mathrm{w} z}=\mu \partial w /\left.\partial y\right|_{y=0}$

Fig. 20 Film thickness distribution as well as contours of the streamwise and spanwise wall shear stress components.

distortion (i.e. change in interface curvature) there. In front of the large wave humps, the region of capillary flow separation is shaped in the form of a horseshoe (in accordance with the shape of capillary wave fronts). This region extends in principle to the capillary wave region of the intermediate hump but is interrupted by a stretch without flow reversal. In addition to the first capillary minimum, flow separation also takes place at the second and third capillary minima. However, this is the case only in the region preceding the intermediate hump.

A more detailed look at capillary flow separation in the regions identified in subfigure 20(b) is provided by Figure 21, which depicts liquid phase velocity components in the region of the first and second capillary minimum. More precisely, subfigures 21(a) to 21(d) display the velocity field within $x-y$-planes at different spanwise positions (highlighted in subfigure 20(b)) between the symmetry planes of the intermediate ( $z=-$ $20 \mathrm{~mm}$ ) and main wave $(z=-10 \mathrm{~mm})$ hump. Since subfigures 21(a) $(z=-$ $20 \mathrm{~mm})$ and $21(\mathrm{~d})(z=-10 \mathrm{~mm})$ correspond to planes of symmetry, the spanwise velocity component there vanishes and streamlines were chosen to represent the velocity field. Conversely, in subfigures 21(b) to 21(c), the spanwise velocity component is illustrated with contours, while the 


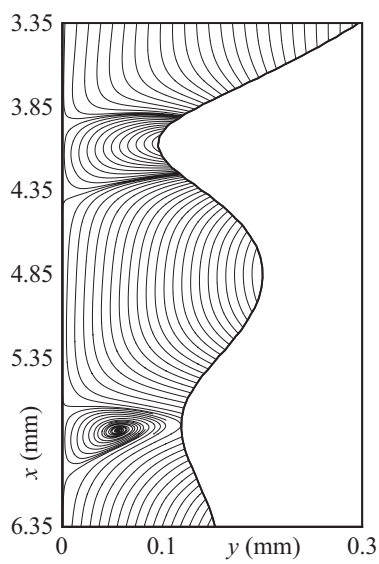

(a) $z=\mathrm{z}_{1}=-20 \mathrm{~mm}$

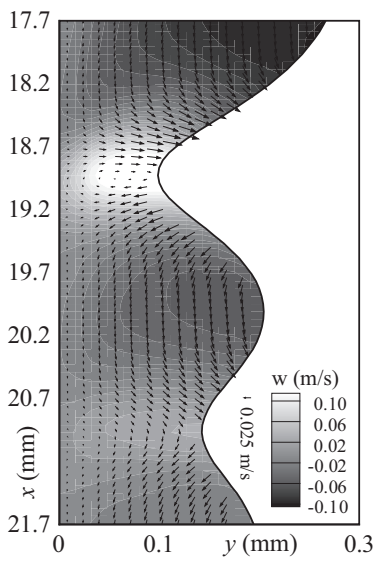

(c) $z=\mathrm{z}_{3}=-12.5 \mathrm{~mm}$

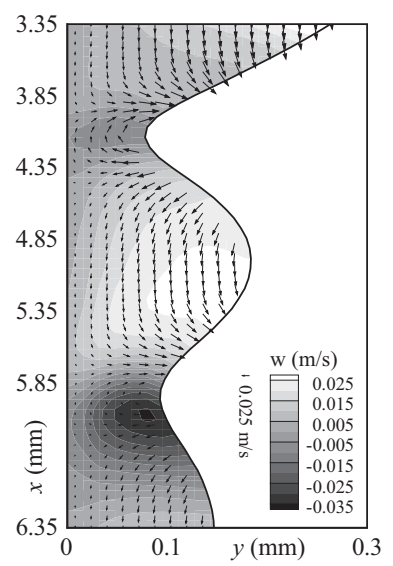

(b) $z=\mathrm{z}_{2}=-18.8 \mathrm{~mm}$

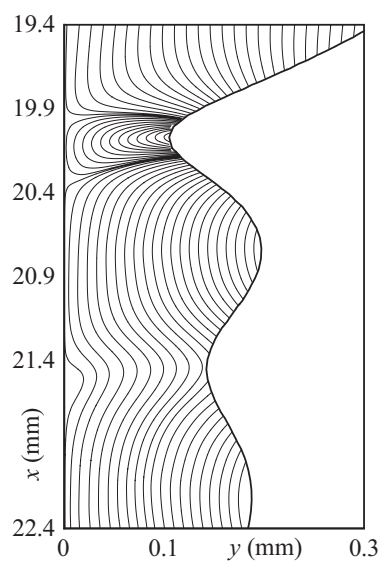

(d) $z=\mathrm{z}_{4}=-10.0 \mathrm{~mm}$

Fig. 21 Simulated streamlines and velocity vectors (projected into $x-y$ planes) near the first two capillary minima at different z-positions (see subfigure 20(b)) for the 3-dimensional film.

streamwise and crosswise components are represented with the help of vectors obtained by projection of the 3-dimensional velocity vector field into the respective planes.

In accordance with the distribution of $\tau_{\mathrm{w} x}$, subfigure 21(a) shows the occurrence of one open and one large closed CSE (associated with the first and second capillary minimum) in front of the intermediate hump at $z=-20 \mathrm{~mm}$. With increasing spanwise distance, the second CSE is seen to disappear at $z=-12.5 \mathrm{~mm}$ (see subfigure $21(\mathrm{c})$ ), where spanwise velocity contours show that the first CSE is associated with strong spanwise flow. This is in accordance with the orientation of the capillary wave front there, which is not horizontal, in contrast to the other spanwise positions, as can be deduced from subfigure 19(b). The occurrence of strong spanwise flow within the CSE suggests a corkscrew-type flow in this region ${ }^{5}$.

In order to gain a topological insight into the 3-dimensional capillary flow separation occurring in the considered water film, Figure 22 depicts liquid phase streamlines in the capillary wave region preceding the intermediate wave hump. Therein, 3-dimensional CSE's are shown to take the form of vortex tubes, the axes of which are shaped like the corresponding capillary wave fronts. Moreover, the "vortex tubes" exhibit corkscrew-shaped streamlines as previously inferred with respect to subfigure 21(c). It should be mentioned here that those parts of streamlines depicted in Figure 22 lying in the gaseous phase were blanked in order to highlight liquid phase conditions. The full streamlines do indeed form

\footnotetext{
${ }^{5}$ Oliver and Atherinos (1968) and Portalski (1964) conjectured (and in the latter case refuted) the existence of such a flow in falling liquid films.
}

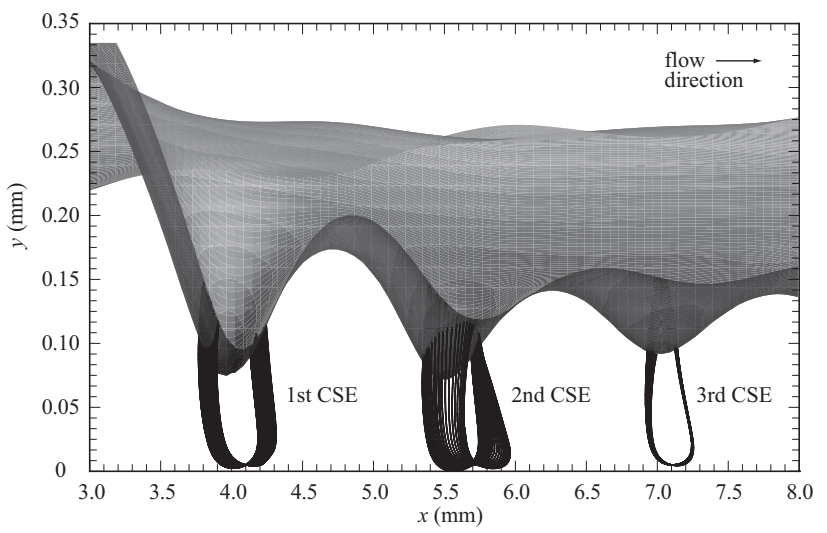

(a) Inner side view (from $z=-20 \mathrm{~mm}$ in negative $z$-direction)

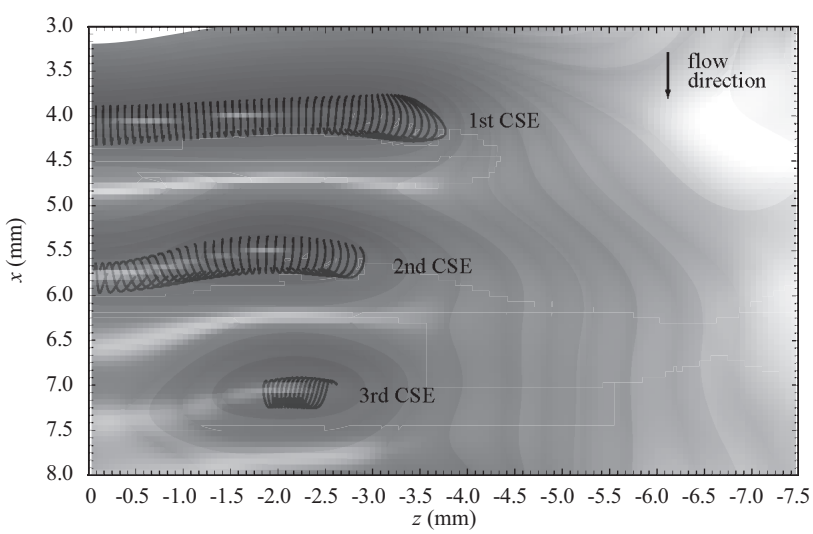

(b) Top view

Fig. 22 Simulated liquid phase streamlines in the capillary wave region of the 3-dimensional film: inner side and top view.

closed "vortex tubes".

A distinctly 3-dimensional capillary feature of the flow is exhibited by the region of capillary interference shown in Figure 18 and subfigure 19(b). Indeed, considering the governing mechanism of capillary flow separation, it can be inferred that the spanwise variation of film thickness associated with the checkerboard pattern there causes spanwise flow. Moreover, since the spanwise component of gravitational acceleration is non existent, it can be expected that this capillary-induced spanwise flow is relatively strong. This is confirmed by the distribution of the spanwise wall shear stress $\tau_{\mathrm{w} z}$ depicted in subfigure 20(c), showing $\tau_{\mathrm{w} z}$ to attain large magnitudes in the interference region. Further, the distribution of $\tau_{\mathrm{w} z}$ clearly shows that the previously discussed interfacial checkerboard pattern is also imposed on the flow.

The occurrence of a checkerboard flow structure raises an interesting prospect from a transport perspective. Indeed, considering a fluid element traveling at a given spanwise position, the latter is subjected to spanwise flow of alternating direction as the region of capillary interference passes over its position. This causes the fluid element to move back and forth in spanwise direction causing strong lateral mixing. In addition, the fluid element is subjected to oscillating streamwise and crosswise velocity components, causing it to adopt a corkscrew-shaped pathline.

In order to investigate the spanwise flow caused by the checkerboard pattern in greater detail, figures 23 and 24 depict liquid phase velocity components within y-z-planes at different streamwise positions. These positions were chosen such that the corresponding planes "cut through" subsequent capillary extrema (and the intermediate hump) as highlighted in subfigure 20(c). In these figures, the streamwise velocity component is 


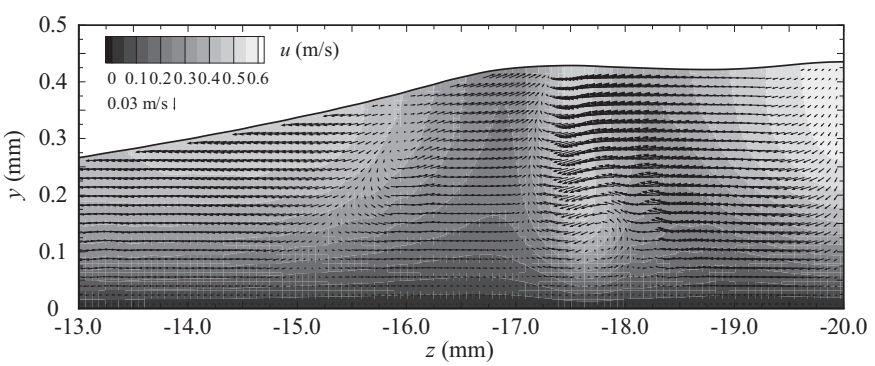

(a) $x=\mathrm{x}_{1}=2.18 \mathrm{~mm}$

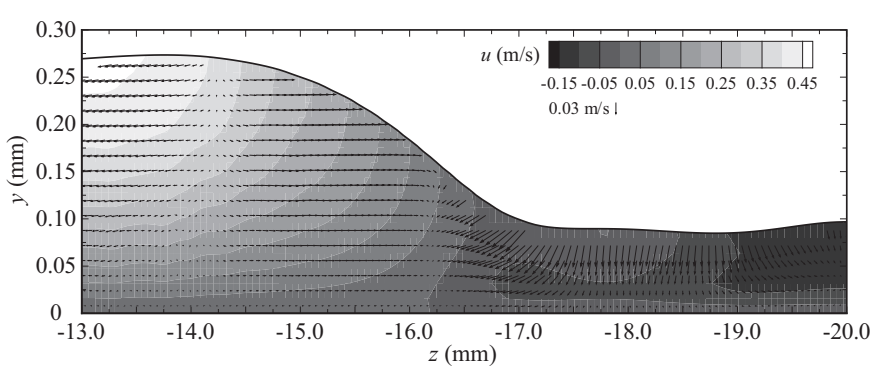

(b) $x=\mathrm{x}_{2}=4.10 \mathrm{~mm}$

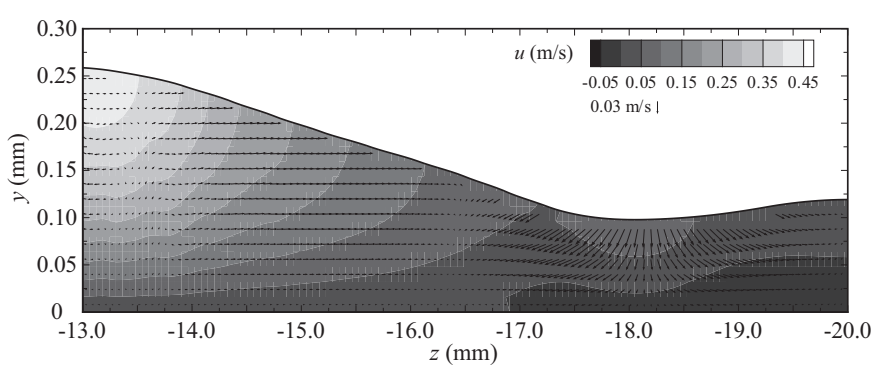

(c) $x=\mathrm{x}_{3}=5.75 \mathrm{~mm}$

Fig. 23 Velocity vectors (projected into $z-y$ planes) at different $x-$ positions (see subfigure 20(c)) in the region of capillary interference.

represented with contours, while spanwise and crosswise velocity components are illustrated with projections of the 3 -dimensional velocity vector field into the respective planes. The most striking feature of the vector plots in these figures is the cellular flow pattern caused by the spanwise variation of film thickness. Indeed, in Figure 24, each capillary wave is associated with two open cells, resembling the open CSE in Figure 21. These cells are associated with strong spanwise flow from the wave humps to the wave troughs. As the position of the latter alternates in spanwise direction, so does the direction of the spanwise flow. Consequently, considering the spanwise position $z=-16.5 \mathrm{~mm}$, a fluid element traveling in the residual layer is subjected to alternating spanwise flow from subfigures 23(a) to 24(c).

Another striking feature of the 3-dimensional flow is seen in subfigure 23(a), representing the velocity field within the intermediate hump. There, between positions $z=-18.25 \mathrm{~mm}$ and $z=-17.25 \mathrm{~mm}$, a "spanwise" eddy is seen to occur. Evidence of this eddy is also discernible at the corresponding position in subfigure 20(c), showing contours of the spanwise wall shear stress. A full investigation of this flow structure's 3dimensional shape as well as its driving mechanism also remains the object of future work.

From a scalar transport perspective, the above discussed intense mixing, caused both by capillary flow separation and the spanwise cellular pattern in the region of capillary interference (as well as the above mentioned "spanwise" eddy), is expected to drastically increase the local driving potential, leading to a significant wave-induced intensification of

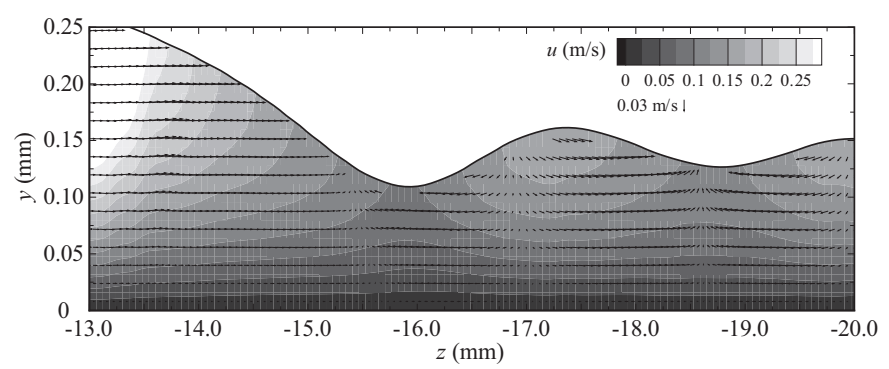

(a) $x=\mathrm{x}_{4}=13.5 \mathrm{~mm}$

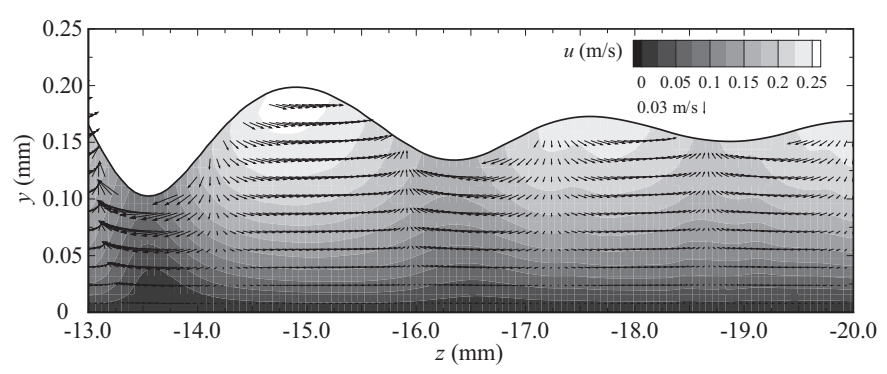

(b) $x=\mathrm{x}_{5}=15.75 \mathrm{~mm}$

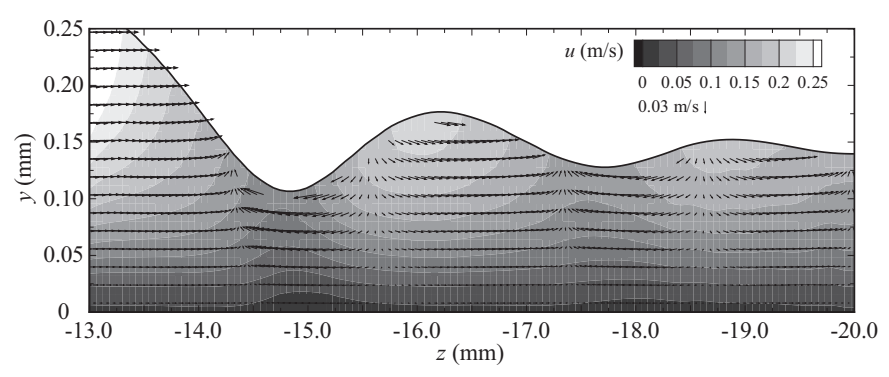

(c) $x=\mathrm{x}_{6}=17.8 \mathrm{~mm}$

Fig. 24 Velocity vectors (projected into z-y planes) at different $x$ positions (see subfigure 20(c)) in the region of capillary interference.

scalar transfer, which could explain the strong increase in transfer rates to 3-dimensional falling liquid films. Finally, the spanwise mixing in the capillary wave region preceding the intermediate hump could exert a stabilizing effect on thermo-capillary film rupture, since it would tend to homogenise the spanwise temperature distribution and thus reduce thermocapillary forces driving film rupture.

\section{CONCLUSIONS}

In this paper, an in-depth investigation of the phenomenon of capillary flow separation, occurring in 2- and 3-dimensional falling liquid films, was presented. The kinematics and governing dynamics of this phenomenon as well as its effect on heat transport were studied based on highly resolved optical film thickness and velocity measurements as well as numerical simulations. The presented results, allow to draw a number of conclusions and recommendations for future research.

Flow separation occurs in the capillary wave region of falling liquid films (preferably at the first capillary minimum) due to the strong streamwise change in curvature of the liquid-gas interface there. This free surface deformation acts on the interfacial pressure jump due to the effect of tensile forces, causing an adverse pressure gradient within the liquid film, which is sufficiently large to compensate streamwise gravitation and induce flow reversal and detachment from the wall. Thereby, the resulting Capillary Separation Eddy or CSE (which only persists over a short time span during the passage of the capillary waves) can either remain closed 
or assume an open shape with streamlines ending at the interface.

In the case of 3-dimensional liquid films, characterized by horseshoeshaped principal wave humps preceded by a region of complex interfering 3-dimensional capillary waves, multiple CSE's occur. These assume the form of vortex tubes, the axes of which follow the corresponding capillary wave fronts. Additionally, in the region of capillary interference, strong spanwise currents (and an associated "spanwise" eddy) occur, which are in principle governed by the same mechanism as the capillary flow separation, i.e. spanwise variations of film thickness causing corresponding pressure variations due to the effect of tensile forces.

These hydrodynamic features of 3-dimensional falling liquid films have yet to be verified experimentally and corresponding investigations are currently under way. In particular, this includes quantitative measurements of the 3-dimensional free surface topology for the flow conditions investigated by Park and Nosoko (2003) (who published "only" qualitative shadowgraphs), using Laser Induced Fluorescence (LIF). Such measurements would enable to confirm the simulated wave topology represented in Figure 20(a). Further, velocity measurements (using LDV and PIV) in the optically accessible RIM test section are envisaged. Due to the necessity to access the film's cross section through the liquid phase (see Figure 4), these measurements are restricted to the residual layer (i.e. $0 \leq y \leq \delta_{\min }$ ) of 3-dimensional liquid films, which however is of most interest in terms of capillary flow separation.

Regarding the effect on heat transport, flow separation causes strong convective transport normal to the wall, which leads to an intensification of wall-side heat transfer. Thereby, the correct mixing length characterizing this process is discernible only in a Lagrangian view of the phenomenon and is considerably smaller than the size of the CSE. Moreover, this mixing length increases with wall distance, suggesting a stronger intensification of interfacial transfer compared to wall-side transfer. $\mathrm{Nu}$ merical simulations of heat transfer at the free surface of falling liquid films (using a VOF-code enabling adaptive grid refinement in the interfacial region) are currently under way in order to verify this conjecture. For the 3-dimensional case, the spanwise flow patterns mentioned above could explain the strong transfer intensification commonly observed in 3-dimensional falling liquid films.

A final recommendation regarding future work on falling liquid films pertains to the elucidation of the relation between liquid phase momentum transport and the film's 2- and 3-dimensional free surface structure. Such insights could be very helpful for the extension of the very successful modelling approaches developed by Ruyer-Quil and Manneville (2000, 2002) and Scheid et al. (2006) (see also Kalliadasis et al. (2011)) as well as Nguyen and Balakotaiah (2000); Mudunuri and Balakotaiah (2006). In particular, the influence of the flow separation phenomenon on large wave dynamics could be of interest. Indeed, Tihon et al. (2006) suggested that capillary waves stabilize the large wave humps they precede. Further, the mechanism leading to the 3-dimensional destabilization of 2-dimensional wavy liquid films needs to be elucidated. This mechanism could be similar to the accelerational Rayleigh-Taylor instability suggested for liquid jets by Marmottant and Villermaux (2004). Alternatively, the Plateau-Rayleigh instability may cause capillary wave fronts to disintegrate in spanwise direction as suggested by Park and Nosoko (2003). This may also explain the more or less uniform spanwise profile of the capillary wave's free surface in subfigures 23(b), which resembles that of a liquid cylinder subject to the Plateau-Rayleigh instability close to the event of fragmentation (see figures 15(c) and 15(d) in Eggers and Villermaux (2008)). However, these conjectures need to be investigated more rigorously. Finally, the full 3-dimensional structure of the "spanwise" eddy observed in subfigure 23(a) remains to be elucidated.

In order to perform the above mentioned investigations, highly resolved numerical simulations of 3-dimensional falling liquid films (preferably over the entire development length) are necessary. For this, the use of a supercomputer is required.

\section{ACKNOWLEDGEMENTS}

The authors gratefully acknowledge the financial support of the Deutsche Forschungsgemeinschaft (DFG) within the Collaborative Research Center (SFB) 540 "Model-based Experimental Analysis of Kinetic Phenomena in Fluid Multi-phase Reactive Systems". The authors also thank S. V. Alekseenko for providing the photograph in figure 1 and F. Al-Sibai for providing the photograph in figure 16(c).

\section{NOMENCLATURE}

$\delta_{\mathrm{Nu}} \quad$ Falling liquid film length scale: $\left(3 q \nu / g_{x}\right)^{1 / 3}$

$u_{\mathrm{Nu}} \quad$ Falling liquid film velocity scale: $q / \delta_{\mathrm{Nu}}$

$\varphi \quad$ Inclination angle: $\arctan \left(g_{x} / g_{y}\right)$

Re Reynolds number of film flow: $u_{\mathrm{Nu}} \delta_{\mathrm{Nu}} / \nu$

$K a \quad$ Kapitza number: $\sigma /\left(\rho g_{x}^{1 / 3} \nu^{4 / 3}\right)$

$W e \quad$ Weber number: $\sigma /\left(\rho \delta_{\mathrm{Nu}} u_{\mathrm{Nu}}^{2}\right)$

$\Psi \quad$ Ratio between pressure and gravitational force acting in streamwise direction on a fluid element at the wall

\section{REFERENCES}

Adomeit, P., Leefken, A., and Renz, U., 2000, "Experimental and numerical investigations on wavy films," E.W.P. Hahne, W. Heidemann, and K. Spindler, editors, Proceedings of the 3rd European thermal sciences conference, 2, 1003-1009, ETS, Heidelberg.

Adomeit, P., and Renz, U., 2000, "Hydrodynamics of three-dimensional waves in laminar falling films," International Journal of Multiphase Flow, 26, 1183-1208. URL http://dx.doi.org/10.1016/S0301-9322(99) 00079-8.

Al-Sibai, F., 1998, Simultane Messung der Strömungs- und Oberflächenstruktur von Rieselfilmen mit zwei- und dreidimensionaler Welligkeit, Master's thesis, RWTH-Aachen University.

Alekseenko, S.V., Antipin, V.A., Guzanov, V.V., Kharlamov, S.M., and Markovich, D.M., 2005, "Three-dimensional solitary waves on falling liquid film at low Reynolds numbers," Physics of Fluids, 17(121704), 14. URL http://dx.doi.org/10.1063/1.2158428.

Alekseenko, S.V., Nakoryakov, V.E., and Pokusaev, B.G., 1994, Wave Flow of Liquid Films, Begell House.

Brackbill, J.U., Kothe, D.B., and Zemach, C., 1992, "A Continuum Method for Modelling Surface Tension," Journal of Computational Physics, 100, 335-354. URL http://dx.doi.org/10.1016/0021-9991(92) 90240-Y.

Brauer, H., 1956, "Strömung und Wärmeübergang bei Rieselfilmen," VDI-Forschungsheft, 457(22), 1-40.

Chang, H.C., Cheng, M., Demekhin, E.A., and Kopelevich, D.I., 1994 , "Secondary and Tertiary Excitation of Three-Dimensional Patterns on a Falling Film," Journal of Fluid Mechanics, 270, 251-275. URL http://dx. doi.org/10.1017/S002211209400426X.

Cohen-Sabban, J., Gaillard-Groleas, J., and Crepin, P.J., 2001, "Quasiconfocal extended field surface sensing," A. Duparre, and B. Singh, editors, Proceedings of SPIE, vol. 4449 of Optical Metrology Roadmap for the Semiconductor, Optical, and Data Storage Industries II, 178-183. URL http://dx.doi.org/10.1117/12.450093.

Demekhin, E.A., Demekhin, I.A., and Shkadov, V.Y., 1983, "Solitons in viscous films flowing down a vertical wall," Fluid Dynamics, 18(4), 500 507. URL http://dx.doi.org/10.1007/BF01090610. 
Demekhin, E.A., Kaplan, M.A., and Shkadov, V.Y., 1987, "Mathematical models of the theory of viscous liquid films," Fluid Dynamics, 22(6), 885-893. URL http://dx.doi.org/10.1007/BF01050727.

Dietze, G.F., 2010, Flow Separation in Falling Liquid Films, Ph.D. thesis, RWTH Aachen University. ISBN 978-3-86844-259-5.

Dietze, G.F., Al-Sibai, F., and Kneer, R., 2009, "Experimental study of flow separation in laminar falling liquid films," Journal of Fluid Mechanics, 637, 73-104. URL http://dx.doi.org/10.1017/S0022112009008155.

Dietze, G.F., Horsky, M., Al-Sibai, F., and Kneer, R., 2011, "Selected Transport Phenomena in Falling Liquid Films," Proceedings of the 5th International Berlin Workshop (IBW5) on Transport Phenomena with Moving Boundaries, vol. 920 of Fortschritt-Berichte VDI Reihe 3, 107-150.

Dietze, G.F., and Kneer, R., 2010, "Capillary flow separation in 2- and 3dimensional laminar falling liquid films," Proceedings of the 14th International Heat Transfer Conference, 753-768, ISBN 978-0-7918-4938-5. URL http://dx.doi.org/10.1115/IHTC14-22064.

Dietze, G.F., Leefken, A., and Kneer, R., 2008, "Investigation of the backflow phenomenon in falling liquid films," Journal of Fluid Mechanics, 595, 435-459. URL http://dx.doi.org/10.1017/S0022112007009378.

Eggers, J., and Villermaux, E., 2008, "Physics of liquid jets," Reports on Progress in Physics, 71, 79. URL http://dx.doi.org/10.1088/0034-4885/ 71/3/036601

Hirt, C.W., and Nichols, B.D., 1981, "Volume of Fluid (VOF) Method for the Dynamics of Free Boundaries," Journal of Computational Physics, 39, 201-225. URL http://dx.doi.org/10.1016/0021-9991(81)90145-5.

Kalliadasis, S., Ruyer-Quil, C., Scheid, B., and Velarde, M.G., 2011, Falling Liquid Films, Springer Verlag (accepted for publication in AMS series).

Kapitza, P.L., 1948, "Wave Flow of Thin Layer of Viscous Fluid (in Russian)," Zhurn Eksper Teor Fiz, 18(1), 3-28.

Kapitza, P.L., and Kapitza, S.P., 1965, "Wave Flow on Thin Layers of a Viscous Fluid," D.T. Haar, editor, Collected papers of P. L. Kapitza, vol. 2, 662-709, Pergamon Press, Oxford.

Kunugi, T., and Kino, C., 2003, "Numerical Simulation of Oscillatory Falling Liquid Film Flows," Fusion Engineering and Design, 65, 387392. URL http://dx.doi.org/10.1016/S0920-3796(03)00007-3.

Kunugi, T., and Kino, C., 2005, "DNS of Falling Film Structure and Heat Transfer via MARS Method," Computers \& Structures, 83(6-7), 455462. URL http://dx.doi.org/10.1016/j.compstruc.2004.08.018.

Kunugi, T., Kino, C., and Serizawa, A., 2005, "Surface Wave Structure and Heat Transfer of Vertical Liquid Film Flow with Artificial Oscillation," 5th International Symposium on Multiphase Flow, Heat Mass Transfer and Energy Conversion, XiŠan.

Leefken, A., and Renz, U., 2001, "Numerical Analysis on Hydrodynamics and Heat Transfer of Laminar-Wavy Falling Films," F.P. Schindler, editor, 1st International Berlin Workshop-IBW1 on Transport Phenomena with Moving Boundaries, vol. 3 of Fortschritt-Berichte VDI, 134143, VDI Verlag, Düsseldorf.

Liu, J., Schneider, J., and Gollub, J.P., 1995, "Three-dimensional instabilities of film flows," Physics of Fluids, 7(1), 55-67. URL http: //dx.doi.org/10.1063/1.868782.

Malamataris, N.A., and Balakotaiah, V., 2008, "Flow Structure Underneath the Large Amplitude Waves of a Vertically Falling Film," AIChE Journal, 54(7), 1725-1740. URL http://dx.doi.org/10.1002/aic.11506.
Malamataris, N.A., Vlachogiannis, M., and Bontozoglou, V., 2002, "Solitary waves on inclined films: Flow structure and binary interactions," Physics of Fluids, 14(3), 1082-1094.

Marmottant, P., and Villermaux, E., 2004, “On spray formation,” Journal of Fluid Mechanics, 498, 73-111. URL http://dx.doi.org/10.1017/ S0022112003006529.

Massot, C., Irani, F., and Lightfoot, E.N., 1966, "Modified Description of Wave Motion in a Falling Film," AIChE Journal, 12(3), 445-455. URL http://dx.doi.org/10.1002/aic.690120311.

Miyara, A., 1999, "Numerical analysis on flow dynamics and heat transfer of falling liquid films with interfacial waves," Heat and Mass Transfer, 35, 298-306. URL http://dx.doi.org/10.1007/s002310050328.

Miyara, A., 2001, "Flow Dynamics and Heat Transfer of Wavy Condensate Film," Journal of Heat Transfer, 123, 492-500. URL http://dx.doi. org/10.1115/1.1370522.

Mudunuri, R.R., and Balakotaiah, V., 2006, "Solitary waves on thin falling films in the very low forcing frequency limit," AIChE Journal, 52(12), 3995-4003. URL http://dx.doi.org/10.1002/aic.11015.

Nakoryakov, V.E., Pokusaev, B.G., Alekseenko, S.V., and Orlov, V.V., 1977, "Instantaneous velocity profile in a wavy fluid film," Journal of Engineering Physics and Thermophysics, 33(3), 1012-1016. URL http: //dx.doi.org/10.1007/BF00860539.

Nguyen, L.T., and Balakotaiah, V., 2000, "Modeling and experimental studies of wave evolution on free falling viscous films," Physics of Fluids, 12(9), 2236-2256. URL http://dx.doi.org/10.1063/1.1287612.

Nosoko, P., Yoshimura, P.N., Nagata, T., and Oyakawa, K., 1996, "Characteristics of Two-Dimensional Waves on a Falling Liquid Film," Chemical Engineering Science, 51(5), 725-732. URL http://dx.doi.org/10.1016/ 0009-2509(95)00292-8.

Oliver, D.R., and Atherinos, T.E., 1968, "Mass transfer to liquid films on an inclined plane," Chemical Engineering Science, 23, 525-536. URL http://dx.doi.org/10.1016/0009-2509(68)89001-3.

Park, C.D., and Nosoko, T., 2003, "Three-Dimensional Wave Dynamics on a Falling Film and associated Mass Transfer," AIChE Journal, 49(11), 2715-2727. URL http://dx.doi.org/10.1002/aic.690491105.

Petviashvili, V.I., and Tsvelodub, D.Y., 1978, "Horseshoe-shaped solitons on an inclined viscous liquid film," Dokl Akad Nauk SSSR, 238, 1321-1323.

Portalski, S., 1964, "Eddy Formation in Film Flow down a Vertical Plate," Industrial \& Engineering Chemistry Fundamentals, 3(1), 49-53. URL http://dx.doi.org/10.1021/i160009a009.

Prandtl, L., 1961, "Über Flüssigkeitsbewegung bei sehr kleiner Reibung," W. Tolmien, H. Schlichting, and H. Görtler, editors, Gesammelte Abhandlungen zur angewandten Mechanik, Hydro- und Aerodynamik, vol. 2, chap. 4, Springer-Verlag, Berlin.

Rastaturin, A., Demekhin, E., and Kalaidin, E., 2006, "Optimal Regimes of Heat-Mass Transfer in a Falling Film," Journal of Non-Equilibrium Thermodynamics, 31, 1-10. URL http://dx.doi.org/10.1515/JNETDY. 2006.001.

Rood, E.P., 1994, "Interpreting Vortex Interactions with a Free Surface," Journal of Fluids Engineering, 116, 91-94. URL http://dx.doi.org/ 35400004991452.0120 
Ruyer-Quil, C., and Manneville, P., 2000, "Improved modeling of flows down inclined planes," The European Physical Journal B, 15(2), 357369. URL http://dx.doi.org/10.1007/s100510051137.

Ruyer-Quil, C., and Manneville, P., 2002, "Further accuracy and convergence results on the modeling of flows down inclined planes by weighted residual approximations," Physics of Fluids, 14(1), 170-183. URL http://dx.doi.org/10.1063/1.1426103.

Salamon, T.R., Armstrong, R.C., and Brown, R.A., 1994, “Traveling waves on vertical films: Numerical analysis using the finite element method," Physics of Fluids, 6, 2202-2220. URL http://dx.doi.org/10. $1063 / 1.868222$.

Schagen, A., Modigell, M., Dietze, G., and Kneer, R., 2006, "Simultaneous measurement of local film thickness and temperature distribution in wavy liquid films using a luminescence technique," International Journal of Heat and Mass Transfer, 49(25-26), 5049-5061. URL http://dx.doi.org/10.1016/j.ijheatmasstransfer.2006.06.010.

Scheid, B., Ruyer-Quil, C., and Manneville, P., 2006, "Wave patterns in film flows: modelling and three-dimensional waves," Journal of Fluid Mechanics, 562, 183-222. URL http://dx.doi.org/10.1017/ S0022112006000978.
Seban, R.A., and Faghri, A., 1978, "Wave effects on the transport to falling laminar liquid films," Journal of Heat Transfer, 100, 143-147. URL http://dx.doi.org/10.1115/1.3450488.

Sychev, V.V., 1998, Asymptotic Theory of Separated Flows, Cambrigde University Press.

Tihon, J., Serifi, K., Argyriadi, K., and Bontozoglou, V., 2006, "Solitary waves on inclined films: their characteristics and the effects on wall shear stress," Experiments in Fluids, 41, 79-89. URL http://dx.doi.org/10.1007/ s00348-006-0158-1.

Tihon, J., Tovchigrechko, V., Sobolik, V., and Wein, O., 2003, "Electrodiffusion detection of the near-wall flow reversal in liquid films at the regime of solitary waves." Journal of Apllied Electrochemistry, 33, 577587. URL http://dx.doi.org/10.1023/A:1024988602276.

Yoshimura, P.N., Nosoko, P., and Nagata, T., 1996, "Enhancement of Mass Transfer into a Falling Laminar Liquid Film by Two-Dimensional Surface Waves-Some Experimental Observations and Modeling," Chemical Engineering Science, 51(8), 1231-1240. URL http://dx.doi.org/10. 1016/0009-2509(95)00387-8. 